\title{
1 A tRNA modification balances carbon and nitrogen metabolism by regulating phosphate
}

\section{2 homeostasis, to couple metabolism to cell cycle progression.}

3 Ritu Gupta ${ }^{1}$, Adhish S. Walvekar ${ }^{1}$, Shun Liang ${ }^{2}$, Zeenat Rashida ${ }^{1,3}$, Premal Shah $^{2 \#}$, Sunil Laxman ${ }^{1 \#}$

$4 \quad{ }^{1}$ Institute for Stem Cell Biology and Regenerative Medicine (inStem)

$5 \quad$ NCBS-TIFR Campus, GKVK Post, Bellary Road Bangalore 560065

$6 \quad{ }^{2}$ Department of Genetics

$7 \quad$ Rutgers University, Piscataway, NJ 08854

$8{ }^{3}$ Manipal Academy of Higher Education, Manipal, Karnataka, 576104, India

$9 \quad$ \#Correspondence: premal.shah@rutgers.edu, sunil@instem.res.in

\section{Abstract}

11 Cells must appropriately sense and integrate multiple metabolic resources to commit to 12 proliferation. Here, we report that cells regulate carbon and nitrogen metabolic homeostasis 13 through tRNA $U_{34}$-thiolation. Despite amino acid sufficiency, tRNA-thiolation deficient cells appear 14 amino acid starved. In these cells, carbon flux towards nucleotide synthesis decreases, and trehalose 15 synthesis increases, resulting in a starvation-like metabolic signature. Thiolation mutants have only 16 minor translation defects. However, these cells exhibit strongly decreased expression of phosphate 17 homeostasis genes, resulting in an effectively phosphate-limited state. Reduced phosphate enforces 18 a metabolic switch, where glucose-6-phosphate is routed towards storage carbohydrates. Notably, 19 trehalose synthesis, which releases phosphate and thereby restores phosphate availability, is central 20 to this metabolic rewiring. Thus, cells use thiolated tRNAs to perceive amino acid sufficiency, and 21 balance carbon and amino acid metabolic flux to maintain metabolic homeostasis, by controlling 22 phosphate availability. These results further biochemically explain how phosphate availability 23 determines a switch to a 'starvation-state'. 


\section{Introduction}

Cells utilize multiple mechanisms to sense available nutrients, and appropriately alter their internal metabolic state. Such nutrient-sensing systems assess internal resources, relay this

27 information to interconnected biochemical networks, and control global responses that collectively 28 reset the metabolic state of the cell, thereby determining eventual cell fate outcomes (Jeong et al., 29 2000; Förster et al., 2003; Zaman et al., 2008; Broach, 2012; Cai and Tu, 2012; Ljungdahl and 30 Daignan-Fornier, 2012) However, much remains unknown about how cells sense and integrate information from multiple nutrient inputs, to coordinately regulate the metabolic state of the cell and commit to different fates.

In this context, the metabolic state of the cell is also closely coupled with protein translation.

Protein synthesis is enormously energy consuming, and therefore must be carefully regulated in tune with nutrient availability (Warner, 2001). Generally, overall translational capacity and output increases during growth and proliferation (Jorgensen et al., 2004), and decreases during nutrient limitation (Wullschleger, Loewith and Hall, 2006). Signaling processes that regulate translational outputs (such as the TORC1 and PKA pathways) are well studied (Wullschleger, Loewith and Hall, 2006; Zaman et al., 2008; Broach, 2012; González and Hall, 2017). Notwithstanding this, little is known about how core components of the translation machinery might directly control metabolic outputs, and thus couple metabolic states with physiological cellular outcomes. required for tRNA folding, stability, or the accuracy and efficiency of translation (Phizicky and Hopper, 2010). However, the roles of many of these highly conserved modifications remain unclear. One such modification is a thiolation of uridine residue present at the wobble-anticodon $\left(U_{34}\right)$ position of specifically glu-, gln- and lys- tRNAs $\left(\mathrm{s}^{2} \mathrm{U}_{34}\right)$ (Gustilo, Vendeix and Agris, 2008; Phizicky and 
and Ncs6, which are evolutionarily conserved (Nakai, Nakai and Hayashi, 2008; Leidel et al., 2009;

Noma, Sakaguchi and Suzuki, 2009). These enzymes incorporate a thiol group derived directly from an amino acid (cysteine), and replace the oxygen present at the 2-position of $U_{34}$ with sulfur (Schmitz et al., 2008; Leidel et al., 2009; Noma, Sakaguchi and Suzuki, 2009). Surprisingly, these thiolated tRNAs appear to have a relatively minor role in general translation, as seen in multiple studies (Rezgui et al., 2013; Zinshteyn and Gilbert, 2013; Klassen et al., 2016; Chou et al., 2017) with modest roles in enhancing the efficiency of wobble base codon-anticodon pairing (Yarian et al., 2002; Rezgui et al., 2013). connection has remained largely unexplored. Thiolated tRNAs are required to maintain metabolic cycles in yeast (Laxman et al., 2013). Further, the amounts of thiolated tRNAs reflect the intracellular availability of sulfur-containing amino acids (cysteine and methionine) (Laxman et al., 2013). Several studies have observed that a loss of this modification alters amino acid homeostasis, inducing the amino-acid starvation regulator Gcn4 (Laxman et al., 2013; Zinshteyn and Gilbert, 2013; Nedialkova and Leidel, 2015). Additionally, studies have found that the loss of tRNA thiolation results in hypersensitivity to oxidative agents, and the TORC1 inhibitor rapamycin (Fichtner et al., 2003; Goehring, 2003; Goehring, Rivers and Sprague, 2003; Laxman and Tu, 2011; Scheidt et al., 2014), all supporting a role for thiolated tRNAs in maintaining metabolic homeostasis. These disparate studies hint that a core function of this tRNA modification may be to sense amino acid availability (primarily methionine/cysteine), and somehow regulate the metabolic state of the cell to appropriately commit to growth. Yet, how thiolated tRNAs regulate metabolism, and the extent to which this may control cellular outcomes remains entirely unaddressed.

In this study, by directly analyzing different metabolic outputs, we identify the metabolic nodes that are altered in tRNA thiolation deficient cells. We find that tRNA thiolation regulates central carbon and nitrogen (amino acid) metabolic outputs, by controlling flux towards storage carbohydrates. In tRNA thiolation deficient cells, overall metabolic homeostasis is altered, with flux 
75 diverted away from the pentose-phosphate pathway/nucleotide synthesis axis, and towards storage

76 carbohydrates trehalose and glycogen. This thereby alters cellular commitments towards growth

77 and cell cycle progression. Counter-intuitively, we discover that this metabolic-state switch in cells

78 lacking tRNA thiolation is achieved by down-regulating a distant metabolic arm of phosphate

79 homeostasis. We biochemically elucidate how regulating phosphate balance can couple amino acid

80 and carbon utilization towards or away from nucleotide synthesis, and identify trehalose synthesis

81 as the pivotal control point for this metabolic switch. Through these findings we show how tRNA

82 thiol-modifications couple amino acid sensing with overall metabolic homeostasis. We further

83 present a general biochemical explanation for how inorganic phosphate homeostasis regulates

84 commitments to different arms of carbon and nitrogen metabolism, thereby determining how cells

85 commit to a 'growth' or 'starvation' state. 
Results

89

Amino acid and nucleotide metabolism are decoupled in tRNA thiolation deficient cells

activation of the amino acid starvation responsive transcription factor Gcn4, in cells lacking tRNA

thiolation. We used prototrophic yeast strain grown in synthetic minimal medium without supplemented amino acids, in order to minimize any possible confounding roles of supplemented amino acids/nucleotides in the medium. We compared relative amounts of amino acids in WT and thiolation mutants by using quantitative, targeted LC-MS/MS approaches. Here, we carefully normalized samples by total cell number (based on optical density at $600 \mathrm{~nm}$ ), as well as biomass. Unexpectedly, we observed a substantial increase in the intracellular pools of amino acids in

107 factor, and is induced upon amino acid starvation (Hinnebusch, 1984, 2005) (Figure 1B). We

108 measured Gcn4 protein amounts in WT and thiolation deficient cells, and contrarily observed 109 increased Gcn4 protein in thiolation mutants (Figure 1C). Further, GCN4 translation was 110 correspondingly higher in thiolation mutants (Figure S1A) (as also seen earlier in (Zinshteyn and 111 Gilbert, 2013; Nedialkova and Leidel, 2015), and this increased GCN4 translation in the thiolation 112 mutants was Gcn2- and elF2 $\alpha$ phosphorylation-dependent (Figure S1B and Figure S1C). These 113 observations comparing actual amino acid amounts in cells with the activity of Gcn4 therefore 
114 present a striking paradox. As canonically understood, Gcn4 is induced upon amino acid starvation,

115 and Gcn4 translation and protein decrease when intracellular amino acid amounts are restored

116 (Hinnebusch, 1984, 2005). Contrastingly, in the results observed here, despite the high amino acid

117 amounts in the tRNA thiolation mutants, the Gcn2-Gcn4 pathway remains induced. We therefore

118 concluded that the metabolic node regulated by tRNA thiolation, resulting in an apparent amino acid

119 starvation signature, cannot be at the level of amino acid biosynthesis and availability.

120 We therefore considered the possible metabolic outcomes of amino acid utilization, and

121 hypothesized that an alteration in amino acid utilization could be a source of this metabolic rewiring.

122 In particular, amino acids are the sole nitrogen donors for de novo nucleotide synthesis (Figure 1D).

123 Since amino acids accumulated in thiolation mutants, we explored the possibility that this was due

124 to reduced de novo nucleotide synthesis. For this, we adopted a metabolic flux based approach to

125 unambiguously estimate new nucleotide synthesis (Walvekar, Rashida, et al., 2018). In such an

126 approach, unlabelled or ${ }^{15} \mathrm{~N}$-labelled ammonium sulfate can be provided as a sole nitrogen source,

127 and label incorporation via glutamine and aspartate into newly formed nucleotides can be

128 measured. Notably, the incorporation of ${ }^{15} \mathrm{~N}$-label into nucleotides (GMP, AMP and CMP) decreased

129 in thiolation mutants relative to WT cells (Figure 1E), indicating reduced flux towards nucleotide

130 synthesis. Consistent with this, we also observed decreased steady-state levels of nucleotides in

131 thiolation mutants (Figure S2A). Notably, the ${ }^{15} \mathrm{~N}$-label incorporation into amino acids (aspartate and

132 glutamine) themselves were not affected in thiolation mutants (Figure S2B), ruling out amino acid

133 synthesis defects. We further addressed this pharmacologically, using a purine-analog, 8-

134 azaadenine, which acts as a pseudo-feedback inhibitor of nucleotide biosynthesis. Consistent with

135 the decreased nucleotide levels observed, thiolation mutants exhibited increased sensitivity to 8-

136 azaadenine (Figure $1 \mathrm{~F}$, and S2C). Collectively, these data show that the loss of tRNA thiolation

137 decreases nucleotide biosynthesis, with a corresponding accumulation of amino acids. Indeed,

138 nucleotide limitation is an established mechanism to induce Gcn4 (Rolfes and Hinnebusch, 1993).

139 We also asked if the decreased nucleotide synthesis was due to reduced expression of nucleotide 
140 biosynthetic genes. We observed that the expression of candidate genes in this pathway were

141 increased in thiolation mutants (Figure S2D), diminishing the possibility of reduced nucleotide

142 biosynthetic capacity as a reason for decreased nucleotides. This is further explored later in this

143 manuscript. Indeed, increased mRNA levels of nucleotide biosynthetic genes observed in thiolation

144 mutants may be due to feedback upregulation in response to reduced nucleotides, which is a well-

145 established phenomenon (Davis and Ares, 2006; Kwapisz et al., 2008).

146 Collectively, despite increased intracellular pools of amino acids, tRNA thiolation deficient

147 cells exhibit signatures of amino acid starvation, including decreased nucleotide biosynthesis. These

148 data show that tRNA thiolation is important for cells to appropriately balance amino acid utilization

149 with nucleotide synthesis.

150

\section{Carbon flux is routed towards storage carbohydrates in thiolation mutants}

152 Despite amino acids being non-limiting in thiolation deficient cells, flux towards nucleotide

153 synthesis was decreased. This observation was in itself puzzling, and the reason was not obvious. We

154 therefore asked if carbon metabolism was instead rewired in the thiolation mutants. Our reasoning

155 was as follows: while amino acids are the sole nitrogen donors for nucleotide synthesis, the carbon

156 backbone for nucleotides is derived from central carbon metabolism (Figure 2A). We reasoned that

157 since the decreased nucleotide synthesis was not due to amino acid limitation, this could instead be

158 due to a metabolic shift where carbon flux is routed away from nucleotide synthesis. Carbon derived

159 from glucose is converted to glucose-6-phosphate, and then is typically directed towards glycolysis

160 and the pentose phosphate pathway (PPP), which provides precursors (ribose sugars) for nucleotide

161 synthesis (Figure 2A) (Boyle, 2005). However, if glucose-6-phosphate is instead diverted towards

162 storage carbohydrates trehalose and glycogen (Figure $2 \mathrm{~A}$ ), this will result in reduced flux into the

163 PPP, and thereby decreased nucleotide synthesis. To assess if this might happen in tRNA thiolation

164 mutants, we pulsed $\left[\mathrm{U}_{-}{ }^{13} \mathrm{C}_{6}\right]$-labelled glucose to growing WT or thiolation deficient cells, and

165 measured label incorporation into different central carbon metabolites, with nucleotides as the end- 
point readout. We observed significantly decreased carbon label incorporation towards new

167 nucleotide synthesis (GMP and AMP) in the thiolation mutants (Figure 2B and S3A). This result is also

168 consistent with the decreased nucleotide synthesis based on amino acid derived nitrogen

169 assimilation, observed earlier (Figure 1E). Note: in this time-frame it is technically difficult to

170 estimate ${ }^{13} \mathrm{C}$-glucose incorporation into glycolytic and pentose phosphate pathway intermediates

171 (2/3-phosphoglycerate, 6-phosphogluconate, ribose-5-P/ribulose-5-P, sedoheptulose-7-P), since the

172 carbon-label incorporation rapidly saturates (Van Heerden et al., 2014). Therefore, as expected,

173 amounts of these labelled metabolites were unchanged in WT and thiolation mutant cells (Figure

174 S3B).

Next, we directly assessed trehalose and glycogen levels biochemically, as the end-point

176 metabolic readouts of the alternative arm where glucose-6-phosphate is diverted away from the

177 PPP. We first estimated the steady-state amounts of trehalose and glycogen with a biochemical

178 assay, and observed a marked increase in these metabolites in the thiolation mutants (Figure $2 \mathrm{C}$ and

$179 \mathrm{~S} 3 \mathrm{C})$. Subsequently, we directly estimated flux towards trehalose synthesis, using $\left[\mathrm{U}-{ }^{13} \mathrm{C}_{6}\right]$-labelled

180 glucose in a similar metabolic flux measurement as described earlier. Here, we observed a strong

181 increase in the synthesis of trehalose $(M+6$ and $M+12$ mass isotopomers) in the tRNA thiolation

182 mutants (Figure 2D). These data together reveal that carbon flux is re-routed in the thiolation

183 mutants towards the storage carbohydrates, and away from nucleotide synthesis. Collectively, these

184 results show that cells lacking tRNA thiolation rewire metabolic outputs towards storage

185 carbohydrates, suggesting a 'starvation-like' metabolic state. Notably, this occurs despite the

186 absence of glucose (carbon) or amino acid (nitrogen) limitation in these cells.

188 tRNA thiolation couples cellular metabolic state with normal cell cycle progression

189 Dissecting physiological roles of such a fine-tuning of metabolic outputs can be challenging,

190 and this has been the case for tRNA thiolation mutants. However, a simple yeast system, termed

191 'yeast metabolic cycles' or metabolic oscillations, has been effective in identifying regulators that 
192 couple metabolism with cell growth/cell-division (Tu et al., 2005; Slavov and Botstein, 2011). In

193 continuous, glucose-limited cultures, yeast cells exhibit robust metabolic oscillations, which are

194 tightly coupled to the cell division cycle, and where DNA replication and cell division are restricted to

195 a single temporal phase (Tu et al., 2005; Chen et al., 2007). In an earlier study we had observed that

196 tRNA thiolation mutants exhibit abnormal metabolic cycles (Laxman et al., 2013). This was

197 reminiscent of phenotypes exhibited by mutants of cell division cycle regulators (Chen et al., 2007).

198 We therefore asked if tRNA thiolation coupled metabolic and cell division cycles. To test this, we

199 sampled the cells at regular intervals of time during the metabolic cycles, and determined their

200 budding index. While WT cells showed synchronized cell cycle progression, tRNA thiolation mutants

201 showed asynchronous cell division (Figure 3A), suggesting a de-coupling of metabolic and cell

202 division cycles. Given our earlier data showing a metabolic rewiring away from nucleotide synthesis

203 in thiolation mutants, we hypothesized that tRNA thiolation controlled normal cell cycle progression

204 by regulating the balance between nucleotide synthesis, and storage carbohydrate synthesis.

211 during the S-phase of the cell cycle, we reasoned that that this S-phase delay is due to decreased flux

212 towards nucleotide synthesis in thiolation mutants. To investigate this, we examined the sensitivity

213 of WT and thiolation deficient cells to hydroxyurea (HU), which inhibits the ribonucleotide reductase

214 (RNR) enzyme and arrests cells in the S-phase. Thiolation mutants exhibited increased sensitivity to

$215 \mathrm{HU}$ (Figure 3E and S4A). This observed HU sensitivity was not due to a defect in the activation of the

216 Rad53 checkpoint pathway in thiolation mutants (Figure S4B). 
218 homeostasis, leading towards regulated nucleotide synthesis, is required for appropriately coupling

219 metabolic state with normal cell cycle progression.

220

221 Loss of tRNA thiolation results in reduced phosphate homeostasis (PHO) related transcripts and

ribosome-footprints

Thus far, it remains unclear why the loss of tRNA thiolation results in this distinct metabolic

224 switch, where carbon and amino acid flux is diverted away from nucleotide synthesis and into

225 storage carbohydrates. This therefore suggests a deeper, non-intuitive regulatory check-point

226 underpinning the overall metabolic rewiring towards a 'starvation-like' state in tRNA thiolation

227 mutants. In order to identify what this controlling bottleneck might be, we identified transcriptional

228 and translational changes in thiolation mutants relative to WT by performing RNA-seq and Ribo-seq

229 (Ingolia et al., 2009) based on methods described in (Weinberg et al., 2016; McGlincy and Ingolia,

230 2017). Ribosome profiling datasets were generated for both distinct thiolation pathway mutants

231 ( $u b a 4 \Delta$ and $n c s 2 \Delta$ ), in three biological triplicates. Figures S5A and S5B show transcript and ribosome

232 footprint read correlations, as well as read-length distributions. Using these datasets, we compared

233 global gene expression, as well as ribosome footprints of WT cells with the $u b a 4 \Delta$ and $n c s 2 \Delta$

234 thiolation mutants (Figure 4A). Notably, comparing WT cells with the thiolation mutants ( $u b a 4 \Delta$ and

$235 n \operatorname{cs} 2 \Delta)$, we find exceptional correlation for transcripts, as well as ribosome footprints $\left(R^{2}>0.97\right.$, and

$236 \mathrm{p}<=2.2 \times 10^{-16}$ for all datasets) (Figure 4A and 4B). These data surprisingly revealed that there are very

237 little gene expression or translation changes observed in the thiolation mutants, with fewer than 30

238 genes up or downregulated at a two-fold change cutoff (arbitrarily used to illustrate the point),

239 compared to WT cells (Figure 4A and 4B). Furthermore, we observe only modest increases in

240 ribosome-densities at codons recognized by thiolated tRNAs - AAA, CAA and GAA in the $u b a 4 \Delta$ and

$241 n c s 2 \Delta$ cells (Figure S5C). Collectively, these extensive analysis show that the loss of tRNA thiolation 
242 has minimal effects on translational outputs in vivo, and any changes observed in the translation

243 rates are likely driven by changes at the transcriptional level.

244 Given the lack of large-scale changes at the transcriptional and translational levels, but

245 robust effects of thiolation mutants on cellular metabolism, we focused on changes in expression

246 levels of genes involved in metabolic pathways. We first examined several general amino acid

247 control (GAAC) response genes, including Gcn4 targets in amino acid biosynthetic pathways, and

248 expectedly found these to be transcriptionally upregulated in the thiolation deficient cells (Figure

249 4C). Additionally, as expected, we observed small increases in GCN4 translation in the thiolation

250 mutants (Figure S6A). These data collectively corroborate our earlier data from Figure 1, and agrees

251 with previous reports (Zinshteyn and Gilbert, 2013; Nedialkova and Leidel, 2015). Also consistent

252 with our earlier data, most nucleotide biosynthesis genes showed an increase in mRNA and

253 ribosome-footprint abundances in the thiolation deficient cells (Figure S6B). In these datasets, there

254 were also no obvious changes in central carbon metabolism genes in thiolation mutants. We also

255 found small decreases in the transcription and translation rates of all large and small subunit genes

256 of ribosomes in $u b a 4 \Delta$ and $n c s 2 \Delta$ cells at the translational level (Figure S6C), consistent with earlier

257 observations (Laxman et al., 2013; Nedialkova and Leidel, 2015).

However, in the course of this extensive functional, we observed that an unusual group of

$\sim 20$ genes were strongly down-regulated in the thiolation mutants, both at the transcript and

based on gene-ontology (GO), we noted that these were functionally related to a metabolic node.

262 These genes are all part of the PHO regulon, which regulates phosphate homeostasis in cells

263 (Ljungdahl and Daignan-Fornier, 2012; Secco et al., 2012). This downregulation of these PHO related

264 genes was exceptionally significant $\left(p<10^{-7}\right)$, compared to other genes across the genome, both at

265 the level of transcript abundances and ribosome-footprints (Figure 4E). Also notably, the unaltered

266 gene transcripts/ribosome footprints in the $\mathrm{PHO}$ regulon (PHO2, PHO4, PHO80, PHO85, PHO87,

267 PHO9O and PHO91) are transcription factors, cyclins/cyclin dependent kinases or low affinity 
phosphate transporters that are not transcriptionally/translationally regulated, but are regulated at

269 the level of their activity (Lemire et al., 1985; Toh-E and Shimauchi, 1986; Madden et al., 1988;

270 Yoshida et al., 1989; Madden, Johnson and Bergman, 1990; Schneider, Smith and O'Shea, 1994;

271 Ogawa et al., 1995; Lenburg and O'Shea, 1996; Auesukaree et al., 2003). Finally, we also observed

272 that some genes related to phospholipid metabolism were downregulated in the thiolation mutants

273 (Figure S6D). Collectively, these data unexpectedly revealed a strong downregulation of genes

274 related to phosphate homeostasis in the tRNA thiolation mutants.

276 Phosphate depletion in wild-type cells phenocopies tRNA thiolation mutants

Inorganic phosphate (Pi) homeostasis is complex, but critical for overall nutrient

278 homeostasis (Ljungdahl and Daignan-Fornier, 2012; Secco et al., 2012). The PHO regulon comprises

279 of several genes that respond to phosphate starvation, and maintains internal phosphate levels by

280 balancing transport of Pi from the external environment, from within vacuolar stores, and the 281 nucleus (Figure 5A) (Ljungdahl and Daignan-Fornier, 2012; Secco et al., 2012). Extensive studies have 282 defined general cellular responses to phosphate limitation (Ogawa, DeRisi and Brown, 2000; Wykoff and O'Shea, 2001; Boer et al., 2003, 2010; Saldanha, Brauer and Botstein, 2004; Gresham et al., 2011; Levy et al., 2011; Choi et al., 2017; Gurvich, Leshkowitz and Barkai, 2017). In general, the PHO observed reduction in $\mathrm{PHO}$ related transcripts and ribosome-footprints in the tRNA thiolation mutants was striking. We therefore first biochemically validated our results from the ribosomeprofiling data. We measured protein amounts of Pho12 and Pho84, two arbitrarily selected PHO genes, in WT cells and the thiolation mutants. These proteins were substantially reduced in $u b a 4 \Delta$ 291 and $n \operatorname{cs} 2 \Delta$ cells (Figure $5 B$ ). This suggests that the thiolation mutants were effectively in a constitutively phosphate-limited state. To test this, we next asked if phosphate starvation resembles 
294 trehalose in WT cells with or without phosphate starvation, and found a robust increase in trehalose

295 after phosphate starvation (Figure 5C). We next measured Gcn4 (protein) in WT cells, with or

296 without phosphate starvation. Here, we observed a strong induction in Gcn4 protein upon

297 phosphate starvation (Figure 5D). Thus, these data from WT cells starved of phosphate strikingly

298 phenocopied the tRNA thiolation mutants. This is also strikingly consistent with earlier metabolic

299 profiling studies of responses to phosphate limitation, which also observed higher amino acid and

300 low nucleotide levels under these conditions (Boer et al., 2010). We therefore conclude that the

301 tRNA thiolation mutants are effectively phosphate-limited, and have altered phosphate

302 homeostasis, due to a reduction in the PHO related genes. This effective phosphate limitation is

303 responsible for the metabolic state switch in these cells.

Trehalose synthesis associated phosphate release enables cells to maintain phosphate balance

This observed downregulation of phosphate metabolism in the thiolation deficient cells is

307 striking. Nonetheless, it is not immediately obvious biochemically how this relates to re-routing

carbon towards storage carbohydrates, and decoupling amino acid metabolism from nucleotide

synthesis. Perplexingly, in our transcript and translation analysis, no other metabolic arms were

similarly decreased in the thiolation deficient cells, and only the amino acid biosynthesis arm

311 (dependent on Gcn4) increases, which we have addressed earlier. Notably, while earlier studies have

312 hinted that phosphate limitation results in a shift towards storage carbohydrates (Lillie and Pringle,

313 1980; Boer et al., 2003, 2010), this more extensive metabolic rewiring has not been carefully

314 analyzed, and a biochemical explanation for this is missing. We wondered if some overlooked

315 biochemical process could explain why a perturbation in phosphate homeostasis connects to the

316 synthesis of storage carbohydrates trehalose and glycogen, as also seen in tRNA thiolation mutants.

317 To address this, we carefully examined all the metabolic nodes altered in the tRNA thiolation

318 mutants, evaluating necessary co-factors and products of each pathway, and looking for possible

319 connections to phosphate. Here, we noted an apparently minor, largely ignored output in the arm of 
carbon metabolism, where glucose-6-phosphate is routed towards trehalose synthesis. The first step

321 of trehalose synthesis is the formation trehalose-6-phosphate (T-6-P), carried out by trehalose-6-

322 phosphate synthase (Tps1). This is followed by the dephosphorylation of T-6-P by Tps2 (Virgilio et al.,

323 1993), forming trehalose (Figure 6A). We noted that this Tps2-dependent second step is

324 accompanied by the release of free, inorganic phosphate (Pi) (Figure 6A). Canonically, these two

325 steps are viewed as an apparently futile trehalose cycle during glycolysis, regenerating glucose, in

326 order to maintain balanced glycolytic flux (van Heerden et al., 2014; Van Heerden et al., 2014).

327 However, we reasoned that if the availability of inorganic phosphate is limiting, a shift to trehalose

328 synthesis can be a way by which cells can liberate $\mathrm{Pi}$, and restore phosphate levels. For this to be

329 generally true, the prediction is that during phosphate starvation, WT cells must accumulate

330 trehalose in order to recover phosphate. As shown earlier, this is exactly what is observed in WT

331 cells limited for phosphate (Figure 5C), and in the tRNA thiolation mutants (Figure 2C and 2D) which

332 are effectively phosphate limited due to a reduction in the $\mathrm{PHO}$ genes.

Given the central role of phosphate, cells utilize all means possible to restore internal

phosphate (Ljungdahl and Daignan-Fornier, 2012). Therefore it is experimentally challenging to study

changes in phosphate homeostasis in cells. However, we directly tested the hypothesis that

trehalose synthesis is a direct way for cells to restore internal phosphate in tRNA thiolation mutants,

337 by utilizing cells lacking TPS2. These cells cannot complete trehalose synthesis, and importantly

338 cannot release phosphate (Figure 6A). We first measured the intracellular Pi levels in WT cells,

339 thiolation mutants $(u b a 4 \Delta)$, cells lacking $\operatorname{Tps} 2 \mathrm{p}(\operatorname{tps} 2 \Delta)$, and cells lacking tRNA thiolation as well as

$340 \operatorname{Tps} 2 \mathrm{p}(u b a 4 \Delta \operatorname{tps} 2 \Delta)$. pho85 $\mathrm{\Delta}$ cells were used as a control, since they exhibit intrinsically higher

341 intracellular Pi levels (Liu et al., 2017). We observed that in cells lacking TPS2 (tps2A) intracellular Pi

342 levels were substantially lower ( 75-80\%) relative to WT cells (Figure 6B and S7A). This suggests that

343 while other pathways (phosphate uptake, glycerol production and vacuolar phosphate export)

344 remain relevant, Tps $2 \mathrm{p}$-mediated Pi release by dephosphorylation of trehalose-6-P is itself important

345 for maintaining internal phosphate levels. Importantly, uba4s cells had only slightly reduced 
346 intracellular Pi levels ( 90\%) relative to WT cells (Figure 6B and S7A). This is consistent with the

347 prediction that due to reduced PHO expression in these cells, they compensate phosphate through

348 increased trehalose synthesis. Contrastingly, the uba4t tps $2 \Delta$ cells showed a dramatic reduction in

349 Pi levels ( 65\%), compared to either of their single mutants. This striking reduction in Pi levels in

350 these cells is consistent with the predicted outcome, where an inability to release phosphate from

351 trehalose (tps $2 \Delta$ ) is also coupled with reduced expression of phosphate assimilation genes (uba4 $\Delta$ ).

352 Next, we tested possible genetic interactions between tps $2 \Delta$ and thiolation mutants ( $u b a 4 \Delta$ and

$353 n \operatorname{cs} 2 \Delta$ ) by assessing relative growth. In our genetic background, tps $2 \Delta$ cells exhibit slightly slower

354 growth at $37^{\circ} \mathrm{C}$. Strikingly, in cells lacking both TPS2 and tRNA thiolation (tps $2 \Delta u b a 4 \Delta$ or tps $2 \Delta$

$355 n c s 2 \Delta$ ), we observed a strong synthetic growth defect, in conditions of low phosphate as well as

356 normal phosphate (Figure 6C and S7B). This is also consistent with the proposed role of Tps2p in

357 maintaining phosphate balance in thiolation mutants. Finally, if we completely imbalance phosphate

358 homeostasis in cells, using cells lacking PHO80, individual mutants of either pho80 $\mathrm{A}$ or thiolation

359 deficient cells show minimal growth defects, but double mutants ( $p h 080 \Delta$ uba $4 \Delta$ or pho80 $\Delta$ cs $2 \Delta$ )

360 show a severe synthetic growth defect (Figure 6D).

361 Collectively, our results suggest that altering phosphate homeostasis by decreasing $\mathrm{PHO}$

362 activity regulates overall carbon and nitrogen flow. Cells deal with decreased phosphate availability

363 by diverting carbon flux away from nucleotide biosynthesis, and towards Tps2-dependent trehalose

364 synthesis and Pi release. This restores phosphate, while concurrently resulting in an accumulation of

365 amino acids, and a reduction in nucleotide synthesis. 
Discussion

In this study, we highlight two related findings- a direct role for a component of translational machinery, $U_{34}$ thiolated tRNAs, in regulating cellular metabolism by controlling phosphate 371 homeostasis; and a biochemical rationale for how phosphate availability regulates flux through 372 carbon and nitrogen metabolism.

A comprehensive model emerges from our studies, explaining how high amounts of thiolated 374 tRNAs reflect a 'growth state', while reduced tRNA thiolation reflect a 'starvation state' (Fig 7). Here, 375 the metabolic control point is phosphate homeostasis. In our proposed model, tRNAs are thiolated 376 in tune with methionine and cysteine availability (Laxman et al., 2013). The presence of these sulfur 377 amino acids reflects an amino acid sufficiency state, suitable for growth (Walvekar, Srinivasan, et al., 378 2018). In these conditions with high amounts of thiolated tRNA, cells thereby direct carbon flux 379 towards nucleotide biosynthesis, coupled with amino acid utilization (as shown in Figs 1 and 2). 380 Accordingly, at this level of metabolic coupling, thiolated tRNAs sense amino acids, and ensure appropriate nucleotide levels for growth and cell cycle progression (as shown in Figs 2 and 3). On the other hand, a reduction in thiolated tRNAs (during methionine/cysteine limitation) (Laxman et al., 2013) switches cells to a 'starvation-like state', by rewiring carbon and nitrogen flux away from nucleotide synthesis and towards storage carbohydrates. This is achieved within the cell by downregulating the $P H O$ regulon, effectively limiting phosphate availability (as shown in Figs 4 and 5). In order to restore phosphate, cells divert glucose flux towards Tps2-mediated trehalose synthesis, and concurrently release $\mathrm{Pi}$ (as shown Fig 5 and 6). Thus, while the trehalose shunt and phosphate recycling restores phosphate levels, this is at the cost of decreased nucleotide biosynthesis, and delayed cell cycle progression. Effectively, the loss of tRNA thiolation rewires cells to a starvation

390 state. Collectively, our study reveals how tRNA thiolation appropriately regulates metabolic outputs 391 by controlling phosphate homeostasis, thereby enabling cells to commit to growth (Fig 7). 392 Intriguingly, this correlation of tRNA thiolation with growth and rewired metabolism is now 
393 emerging in cancer development (McMahon and Ruggero, 2018; Rapino et al., 2018), suggesting

394 possibly conserved metabolic roles for these modified tRNAs.

A modified tRNA is an unusual but effective mechanism to coordinately regulate metabolic

homeostasis. Notably, while several previous studies have observed decreased phosphate-related al., 2017), any possible role for phosphate in tRNA thiolation mediated function has been ignored.

399 Furthermore, tRNAs undergo four conserved modifications in the $U_{34}$ position: 5400 methoxycarbonylmethyluridine $\left(\mathrm{mcm}^{5} \mathrm{U}_{34}\right)$, 2-thiouridine $\left(\mathrm{s}^{2} \mathrm{U}_{34}\right)$, 5-methoxycarbonylmethyl-2401 thiouridine $\left(\mathrm{mcm}^{5} \mathrm{~s}^{2} \mathrm{U}_{34}\right), 5$-methylaminomethyluridine $\left(\mathrm{mnm}^{5} \mathrm{U}_{34}\right)$ (refs). Here we focus only on how the $s^{2} U_{34}$ modification (which is derived from sulfur amino acids) regulates cellular metabolic state. Interestingly, the other $U_{34}$ modifications all require s-adenosyl methionine (SAM), and SAM is 404 directly derived from sulfur amino acid metabolism (Thomas and Surdin-Kerjan, 1997). Also interestingly, mutants of all these related $U_{34}$ tRNA modifications show similar metabolic phenotypes as the thiolation mutants (Zinshteyn and Gilbert, 2013; Nedialkova and Leidel, 2015; Chou et al., 407 2017; Han et al., 2018), and have altered phosphate homeostasis (Chou et al., 2017), raising the 408 possibility that these $U_{34}$-tRNA modifications use similar mechanisms to regulate metabolic 409 homeostasis. A primordial role of $U_{34}$-tRNA modifications might therefore be to sense amino acid 410 sufficiency, and control metabolic state switching towards growth, regulating phosphate availability 411 as a means to achieve this. Co-opting tRNAs (which are the translation components most closely 412 linked to amino acids) to control metabolic states can therefore be an efficient means to ensure appropriate commitments to growth and proliferation, and maximize cellular fitness. extent of carbon and nitrogen flux towards nucleotide synthesis. While it is textbook knowledge that 416 inorganic phosphate is important for glucose homeostasis (Mason et al., 1981; Boyle, 2005; van 417 Heerden et al., 2014; Van Heerden et al., 2014), the biochemical connection of phosphate balance to 
418 carbon and nitrogen flux remains poorly explained. Studies have long observed that trehalose

419 increases upon phosphate starvation (Lillie and Pringle, 1980; Klosinska et al., 2011), and TPS2 is also

420 upregulated (Ogawa, DeRisi and Brown, 2000). In these conditions, central carbon metabolism is

421 down, and phosphate limitation is a 'general starvation' cue (Brauer et al., 2008; Boer et al., 2010;

422 Gurvich, Leshkowitz and Barkai, 2017). Why this occurs has not been immediately apparent. Our

423 study, identifying the trehalose shunt as a way to restore phosphate balance, explains these

424 observations. These data also biochemically explain earlier observations from pathogenic fungi,

425 which show that that the amount of trehalose synthesis determines flux through the pentose

426 phosphate pathway, and nitrogen metabolism (Wilson et al., 2007). Additionally, a recent study

427 observed that phosphate starvation results in not just better cell survival in limited nutrient

428 conditions, but also promotes efficient recovery when the nutrients become available (Gurvich,

429 Leshkowitz and Barkai, 2017). Since trehalose accumulation and utilization is tightly coupled with

430 exit and re-entry into cell division cycle respectively (Shi et al., 2010; Shi and Tu, 2013), we propose

431 that trehalose synthesis during phosphate-limitation has dual roles. Trehalose synthesis concurrently

432 releases inorganic phosphate, which restores phosphate balance in the cell and diverts flux away

433 from nucleotide synthesis and growth. When phosphate is no longer limiting, cells can liquidate

434 trehalose to re-enter the cell division cycle, enabling rapid recovery.

437 phosphate homeostasis. More generally, we biochemically explain how phosphate homeostasis

438 determines flux through different arms of carbon and nitrogen metabolism.

\section{Experimental Procedures}

\section{Yeast strains, media and growth conditions}


442 The prototrophic CEN.PK strain of Saccharomyces cerevisiae was used in all the experiments (Van

443 Dijken et al., 2000). All the strains used in this study are listed in Table S1. For all experiments, cells

444 were grown overnight at $30^{\circ} \mathrm{C}$ in rich media ( $1 \%$ yeast extract, $2 \%$ peptone, $2 \%$ dextrose), washed

445 once and subsequently sub-cultured in minimal media $(0.67 \%$ yeast nitrogen base without amino

446 acids, $0.1 \%$ glucose) unless specified. Phosphate-limited media was prepared as described previously

447 (Klosinska et al., 2011) except that $0.1 \%$. glucose was used unless specified. The only source of

448 phosphorus in phosphate-limited media (low $\mathrm{Pi}$ ) was $\mathrm{KH}_{2} \mathrm{PO}_{4}$, which was present at a concentration

449 of $0.15 \mathrm{mM}$. In high phosphate media (high $\mathrm{Pi}$ ), $\mathrm{KH}_{2} \mathrm{PO}_{4}$ was present at a concentration of $7.5 \mathrm{mM}$

450 with $0.1 \%$. glucose unless specified. In no phosphate media (no $\mathrm{Pi}$ ), $\mathrm{KH}_{2} \mathrm{PO}_{4}$ was completely absent

451 and $0.1 \%$. glucose was used.

452

\section{Western blot analysis}

454 For Gcn4, Pho12 and Pho84 protein levels, cells were grown overnight in rich media, washed once

455 and subsequently sub-cultured in minimal media at an initial $\mathrm{OD}_{600}$ of 0.1 and grown till the $\mathrm{OD}_{600}$ reaches 0.8-1.0. For Gcn4 protein levels in high and no Pi media, cells were grown overnight in rich media, washed once and subsequently sub-cultured in either high Pi or no Pi media at an initial

$460400 \mu \mathrm{l}$ of $10 \%$ trichloroacetic acid and lysed by bead-beating three times. The precipitates were 461 collected by centrifugation, resuspended in $400 \mu$ l of SDS-glycerol buffer (7.3\% SDS, 29.1\% glycerol 462 and $83.3 \mathrm{mM}$ Tris base) and heated at $100^{\circ} \mathrm{C}$ for $10 \mathrm{~min}$. The lysate was cleared by centrifugation 463 and protein concentration was determined by using a bicinchoninic acid assay (23225, Thermo 464 Fisher). Equal amounts of samples were electrophoretically resolved on $4-12 \%$ pre-cast Bis-tris 465 polyacrylamide gels (NP0322BOX, Invitrogen). Anti-HA (12CA5, Roche) and anti-FLAG (F1804-5MG, 
467 proteins. Horseradish peroxidase-conjugated secondary antibodies (mouse and rabbit) were

468 obtained from Sigma-Aldrich. For Western blotting, standard enhanced chemiluminescence reagent

469 (GE Healthcare) was used. Coomassie brilliant blue R-250 was used to stain gels for loading control.

471 Metabolite extraction and LC-MS/MS analysis

472 For steady state amino acids levels, cells were grown overnight in rich media, washed once and

473 subsequently sub-cultured in minimal media at an initial $\mathrm{OD}_{600}$ of 0.1 and grown till the $\mathrm{OD}_{600}$

474 reaches $0.8-1.0 . \sim 10 \mathrm{OD}_{600}$ cells were quenched with $60 \%$ methanol at $-40^{\circ} \mathrm{C}$, and metabolites were

475 extracted, as explained in detail (Walvekar, Rashida, et al., 2018). For ${ }^{15} \mathrm{~N}$-label incorporation in

476 amino acids and nucleotides, cells were grown overnight in rich media, washed once and

477 subsequently sub-cultured in minimal media ( $0.67 \%$ yeast nitrogen base without amino acids and

478 ammonium sulfate, $0.1 \%$ glucose, $20 \mathrm{mM}$ ammonium sulfate) at an initial $\mathrm{OD}_{600}$ of 0.1 and grown till

479 the $\mathrm{OD}_{600}$ reaches $0.5 .{ }^{15} \mathrm{~N}_{2}$-ammonium sulfate (299286, Sigma-Aldrich) was added to reach a ratio of

$48050 \%$ unlabeled to $50 \%$ fully labeled ammonium sulfate. Metabolites were extracted from $\sim 6 \mathrm{OD}_{600}$

481 cells. For ${ }^{13} \mathrm{C}$ - label incorporation in nucleotides and other central carbon metabolites, cells were

482 grown overnight in rich media, washed once and subsequently sub-cultured in minimal media at an

483 initial $\mathrm{OD}_{600}$ of 0.1 and grown till the $\mathrm{OD}_{600}$ reaches 0.5 . $\left[\mathrm{U}-{ }^{13} \mathrm{C}_{6}\right]$ glucose $(\mathrm{CLM}-1396-\mathrm{PK}$, Cambridge

484 Isotope Laboratories) was added to reach a ratio of $50 \%$ unlabeled to $50 \%$ fully labeled glucose.

485 Metabolites were extracted from $\sim 6 \mathrm{OD}_{600}$ cells. Extensive metabolite extraction protocols are

486 described (Walvekar, Rashida, et al., 2018). Metabolites were analyzed using LC-MS/MS method as

487 described (Walvekar et al, 2018). Standards were used for developing multiple reaction monitoring

488 (MRM) methods on Thermo Scientific TSQ Vantage Triple Stage Quadrupole Mass Spectrometer or

489 Sciex QTRAP 6500. All the parent/product masses relevant to this study are listed in Table S3. Amino

490 acids were detected in the positive polarity mode. For nucleotide measurements, nitrogen base

491 release was monitored in the positive polarity mode. Trehalose was detected in the negative polarity 
492 mode. For PPP metabolites and other triose phosphates, phosphate release was monitored in the

493 negative polarity mode.

494 Metabolites were separated using a Synergi $4 \mu$ Fusion-RP 80 A column $(100 \times 4.6 \mathrm{~mm}$, Phenomenex)

495 on Agilent's 1290 infinity series UHPLC system coupled to the mass spectrometer. For positive

496 polarity mode, buffers used for separation were- buffer $\mathrm{A}$ : $99.9 \% \mathrm{H}_{2} \mathrm{O} / 0.1 \%$ formic acid and buffer B:

$49799.9 \%$ methanol/0.1\% formic acid (Column temperature, $40^{\circ} \mathrm{C}$; Flow rate, $0.4 \mathrm{ml} / \mathrm{min} ; \mathrm{T}=0 \mathrm{~min}, 0 \%$

$498 \mathrm{~B} ; \mathrm{T}=3 \mathrm{~min}, 5 \% \mathrm{~B} ; \mathrm{T}=10 \mathrm{~min}, 60 \% \mathrm{~B} ; \mathrm{T}=11 \mathrm{~min}, 95 \% \mathrm{~B} ; \mathrm{T}=14 \mathrm{~min}, 95 \% \mathrm{~B} ; \mathrm{T}=15 \mathrm{~min}, 5 \% \mathrm{~B} ; \mathrm{T}=16$

$499 \min , 0 \% \mathrm{~B} ; \mathrm{T}=21 \mathrm{~min}$, stop). For negative polarity mode, buffers used for separation were- buffer A:

$5005 \mathrm{mM}$ ammonium acetate in $\mathrm{H}_{2} \mathrm{O}$ and buffer $\mathrm{B}$ : $100 \%$ acetonitrile (Column temperature, $25^{\circ} \mathrm{C}$; Flow

501 rate: $0.4 \mathrm{ml} / \mathrm{min} ; \mathrm{T}=0 \mathrm{~min}, 0 \% \mathrm{~B} ; \mathrm{T}=3 \mathrm{~min}, 5 \% \mathrm{~B} ; \mathrm{T}=10 \mathrm{~min}, 60 \% \mathrm{~B} ; \mathrm{T}=11 \mathrm{~min}, 95 \% \mathrm{~B} ; \mathrm{T}=14 \mathrm{~min}$,

$50295 \% \mathrm{~B} ; \mathrm{T}=15 \mathrm{~min}, 5 \% \mathrm{~B} ; \mathrm{T}=16 \mathrm{~min}, 0 \% \mathrm{~B} ; \mathrm{T}=21 \mathrm{~min}$, stop). The area under each peak was

503 calculated using Thermo Xcalibur software (Qual and Quan browsers) and AB SCIEX MultiQuant

504 software 3.0.1.

505

506 Spotting assay for comparative cell growth estimation

507 For all spotting assays, cells were grown overnight in rich media, washed once and subsequently

508 sub-cultured in minimal media at an initial $\mathrm{OD}_{600}$ of $0.2-0.25$ and grown till the $\mathrm{OD}_{600}$ reaches 0.8-1.0.

509 Cells were harvested by centrifugation, washed once with water and $10 \mu \mathrm{l}$ sample for each

510 suspension was spotted in serial 10 -fold dilutions. For 8-aza adenine and hydroxyurea sensitivity

511 assays, cells were spotted onto minimal media plates containing 250 and $300 \mu \mathrm{g} / \mathrm{ml} 8$-aza adenine

512 (A0552, TCl chemicals) or $150 \mathrm{mM}$ hydroxyurea (H8627, Sigma-Aldrich) and incubated at $30^{\circ} \mathrm{C}$. For

513 control plates without drug, images were taken after 1-2 days and for drug containing plates after 4-

5145 days. For genetic interaction analysis with Tps2, cells were spotted onto high and low Pi media

515 plates with $2 \%$ glucose. Plates were incubated at $30^{\circ} \mathrm{C}$ and $37^{\circ} \mathrm{C}$. For genetic interaction analysis 
516 with Pho80, cells were spotted onto minimal media plates with $0.1 \%$ and $2 \%$ glucose. Plates were

517 incubated at $30^{\circ} \mathrm{C}$.

518

519 Trehalose and glycogen measurements

520 For trehalose and glycogen measurements in wild-type and thiolation mutants, cells were grown

521 overnight in rich media, washed once and subsequently sub-cultured in minimal media at an initial

$522 \mathrm{OD}_{600}$ of 0.1 and grown till the $\mathrm{OD}_{600}$ reaches 0.8 -1.0. For trehalose measurement in high and no $\mathrm{Pi}$

523 media, cells were grown overnight in rich media, washed once and subsequently sub-cultured in

524 either high or no Pi media at an initial $\mathrm{OD}_{600}$ of 0.1 and incubated for 8 hours at $30^{\circ} \mathrm{C}$. Cells were

525 harvested by centrifugation and washed with ice-cold water. Cells were lysed in $0.25 \mathrm{M}$ sodium

526 carbonate by incubating at $95-98^{\circ} \mathrm{C}$ for 4 hours. Subsequently, added $0.15 \mathrm{ml} 1 \mathrm{M}$ acetic acid and 0.6

$527 \mathrm{ml}$ of $0.2 \mathrm{M}$ sodium acetate to bring the solution to $\mathrm{pH}$ 5.2. Trehalose and glycogen were digested

528 overnight using trehalase (T8778, Sigma-Aldrich) and amyloglucosidase (10115, Sigma-Aldrich)

529 respectively. Glucose released from these digestions was measured using a Glucose (GO) Assay Kit

530 (GAGO20, Sigma-Aldrich). The concentration of released glucose $(\mu \mathrm{g} / \mathrm{ml})$ was determined from the

531 standard curve and plotted. Statistical significance was determined using Student $T$-test (GraphPad

532 Prism 7).

533

534 Phosphate measurement

535 Cells were grown overnight in rich media, washed once and subsequently sub-cultured in minimal

536 media at an initial $\mathrm{OD}_{600}$ of 0.1 and grown till the $\mathrm{OD}_{600}$ reaches $0.8-1.0$. Free intracellular phosphate

537 levels were determined as described (McNaughton et al., 2010). Briefly, cells were harvested by

538 centrifugation and washed twice with ice-cold water. Cells were lysed by resuspending in $200 \mu l$

$5390.1 \%$ triton $\mathrm{X}-100$ and vortexed for $5 \mathrm{~min}$ with glass beads. The lysate was cleared by centrifugation 
540 and protein concentration was determined by using bicinchoninic acid assay (23225, Thermo

541 Fisher). $30 \mu \mathrm{g}$ of whole cell lysate was used for measurement of free intracellular phosphate levels

542 using ammonium molybdate and ascorbic acid colorimetric assay as described (Ames, 1966).

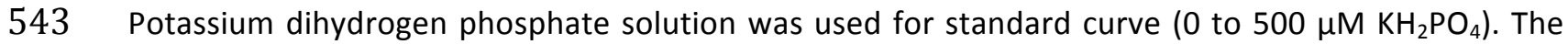

544 amount of phosphate was expressed as $\mu \mathrm{M}$ Pi. Statistical significance was determined using a

545 Student $T$-test (GraphPad Prism 7).

546

\section{Cell cycle synchronization and flow cytometry analysis}

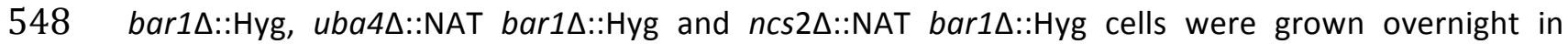
549 minimal media and subsequently sub-cultured in minimal media at an initial $\mathrm{OD}_{600}$ of 0.05 and grown 550 till the $\mathrm{OD}_{600}$ reaches 0.2 . Cells were harvested by centrifugation, washed with water and 551 resuspended in the same medium containing $10 \mu \mathrm{g} / \mathrm{ml}$ of $\alpha$-factor (GenScript). Cells were kept at $55230^{\circ} \mathrm{C}$ for 3 hours till complete $\mathrm{G} 1$ arrest was observed by light microscopy. Subsequently, $5 \mathrm{ml}$ 553 culture was harvested by centrifugation, washed with water and fixed with $70 \%$ ethanol for G1554 arrested population. Remaining culture was synchronously released into the cell cycle by washing 555 away the $\alpha$-factor. Cells were collected at different intervals of time post G1 release, fixed with 556 ethanol, treated with RNaseA (R4875, Sigma-Aldrich) and a protease solution (P6887, Sigma-Aldrich) 557 as described (Haase and Reed, Cell cycle, 2002). Cells were stained with SYTOX green (S7020, 558 Invitrogen) and analyzed on BD FACS Verse flow cytometer.

\section{Time-lapse live cell microscopy}

560 Cells were grown overnight in rich media, washed once and subsequently sub-cultured in minimal

561 media at an initial $\mathrm{OD}_{600}$ of 0.1 and grown till the $\mathrm{OD}_{600}$ reaches $0.4-0.5 .1 .5 \%$ agar pads $(50081$, 562 Lonza) were prepared containing minimal media. The pad was cut into small pieces after it solidified.

$5632 \mu \mathrm{l}$ of the cell suspension was placed on the agar pad, which was inverted and placed in a glass 
564 bottom confocal dish (101350, SPL Life Sciences) for imaging. Phase-contrast images were captured

565 after every 3 minutes' interval for total of 360 mins on ECLIPSE Ti2 inverted microscope (NIKON) and

566 60X oil-immersion objective. Images were stacked and analyzed using ImageJ software. Statistical

567 significance was determined using a Student $T$-test (GraphPad Prism 7).

Transcriptome and Ribosome profiling analyses

570 Cells were grown overnight in rich media and subsequently shifted to minimal media till the OD600

571 reaches $0.5-0.8$. Cells were rapidly harvested by filtration and lysed, as described in detail (Weinberg

572 et al., 2016; McGlincy and Ingolia, 2017). For both transcriptome and ribosome profiling analyses,

573 three biological replicates each for WT and tRNA thiolation mutant cells ( $u b a 4 \Delta$ and ncs $2 \Delta$ ) were

574 included. Total RNA and ribosome-protected fragments were isolated from the cell lysates and RNA-

575 seq and ribosome profiling were performed, as described (Weinberg et al., 2016; McGlincy and

576 Ingolia, 2017), with minor modifications. Separate $5^{\prime}$ and $3^{\prime}$ linkers were ligated to the RNA-

577 fragment instead of $3^{\prime}$ linker followed by circularization (Subtelny et al., 2014). $5^{\prime}$ linkers contained 4

578 random nt unique molecular identifier (UMI) similar to a $5 \mathrm{nt}$ UMI in $3^{\prime}$ linkers. During size-selection,

579 we restricted the footprint lengths to 18-34 nts. Matched RNA-seq libraries were prepared using

580 RNA that was randomly fragmentation by incubating for $14 \mathrm{~min}$ at $95 \mathrm{C}$ with in $1 \mathrm{mM}$ EDTA, $6 \mathrm{mM}$

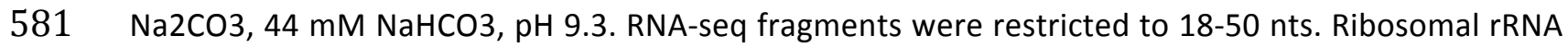

582 were removed from pooled RNA-seq and footprinting samples using RiboZero (Epicenter MRZH116).

583 cDNA for the pooled libraries were PCR amplified for 16 cycles.

\section{Ribosome profiling data processing and analysis}

585 RNA-seq and footprinting reads were mapped to the yeast transcriptome using the riboviz pipeline

586 (Carja et al., 2017). Sequencing adapters were trimmed from reads using Cutadapt 1.14 (Martin,

587 2011) (--trim-n -e 0.2 -minimum-length 24). The reads from different samples were separated based 
on the barcodes in their $3^{\prime}$ linkers using fastx_barcode_splitter (FASTX toolkit, Hannon lab) with utmost one mismatch allowed. UMI and barcodes were removed from reads in each sample using Cutadapt (--trim-n -m $10-\mathrm{u} 4-\mathrm{u}-10)$. Trimmed reads that aligned to yeast rRNAs and tRNAs were removed using HISAT2 v2.1.0 (Kim, Langmead and Salzberg, 2015). Remaining reads were mapped to a set of 5,812 genes in the yeast genome (SGD version R64-2-1_20150113) using HISAT2. Only reads and gff files were obtained from riboviz package (https://github.com/shahpr/RiboViz, (Carja et al., 2017). Gene-specific fold-changes in RNA and footprint abundances were estimated using DESeq2 packages in R (Love, Huber and Anders, 2014) using default log-fold-change shrinkage options.

597 Changes in ribosome-densities (translation efficiencies) were estimated using the Riborex package in 598 R (Li et al., 2017).

599 The complete transcript/ribosome footprint datasets are available at GEO (number GSE124428).

600 Link: https://www.ncbi.nIm.nih.gov/geo/query/acc.cgi?acc=GSE124428

601

Continuous chemostat culture growth to study yeast metabolic cycles, and microscopic analysis

603 Continuous chemostat cultures to establish the YMC were performed as described previously (Tu et

604 al, Science, 2005). An overnight batch culture of prototrophic CEN.PK strain (van Dijken et al, Enzyme 605 Microb Technol, 2000) grown in rich medium was used to inoculate working volume of $1 \mathrm{~L}$ in the 606 chemostat. At 20-minute time-intervals, cells were fixed with $2 \%$ paraformaldehyde, and imaged 607 under a bright-field microscope. $\sim 200$ cells from each time point were sampled, and budding cells 608 were counted manually.

610 Author abbreviations: RG and SLa conceived the project. RG, PS and SLa designed the study. RG, 611 ASW, SL and ZR performed experiments. RG and SL prepared RNA-seq and ribosome-profiling 
612 libraries. PS performed detailed analysis of RNA-seq and ribosome-profiling data. RG and SL wrote

613 the manuscript, with contributions from the other authors.

614 Note: SL (Shun Liang), Sla (Sunil Laxman).

615

616 Acknowledgements

617 We acknowledge the extensive use of the NCBS/inStem/CCAMP mass spectrometry facility for LC-

$618 \mathrm{MS} / \mathrm{MS}$ instrument support. We also thank Dr. Anjana Badrinarayan for the use of her live-cell

619 imaging microscope system. We thank Claudio de Virgilio, Nikolai Slavov, Sider Penkov, and Sriram

620 Varahan for critical comments on this manuscript. RG and ASW are supported by the Department of

621 Science and Technology and Science and Engineering Research Board (DST-SERB) national

622 postdoctoral fellowships (PDF/2016/000416 and PDF/2015/000225 respectively). SLa is supported

623 by an Intermediate Fellowship from the Wellcome Trust-DBT India Alliance (grant number

$624 \mathrm{IA} / \mathrm{I} / 14 / 2 / 501523)$, as well as institutional support from inStem and the Dept. of Biotechnology

625 (Govt. of India). PS is supported by grants NIH R35 GM124976 and start-up funds from the Human

626 Genetics Institute of New Jersey at Rutgers University

627

628 
630 Ames, B. N. (1966) '[10] Assay of inorganic phosphate, total phosphate and phosphatases', Methods

631 in Enzymology. doi: 10.1016/0076-6879(66)08014-5.

632 Auesukaree, C. et al. (2003) 'Transcriptional regulation of phosphate-responsive genes in low-affinity

633 phosphate-transporter-defective mutants in Saccharomyces cerevisiae', Biochemical and Biophysical

634 Research Communications. doi: 10.1016/S0006-291X(03)01068-4.

635 Björk, G. R. et al. (1987) 'Transfer RNA modification.', Annual review of biochemistry. doi:

636 10.1146/annurev.biochem.56.1.263.

637 Boer, V. M. et al. (2003) 'The genome-wide transcriptional responses of Saccharomyces cerevisiae

638 grown on glucose in aerobic chemostat cultures limited for carbon, nitrogen, phosphorus, or sulfur',

639 Journal of Biological Chemistry. doi: 10.1074/jbc.M209759200.

640 Boer, V. M. et al. (2010) 'Growth-limiting Intracellular Metabolites in Yeast Growing under Diverse

641 Nutrient Limitations', Molecular Biology of the Cell. doi: 10.1091/mbc.E09-07-0597.

642 Boyle, J. (2005) 'Lehninger principles of biochemistry (4th ed.): Nelson, D., and Cox, M.',

643 Biochemistry and Molecular Biology Education. doi: 10.1002/bmb.2005.494033010419.

644 Brauer, M. J. et al. (2008) 'Coordination of Growth Rate, Cell Cycle, Stress Response, and Metabolic

645 Activity in Yeast', Molecular Biology of the Cell. doi: 10.1091/mbc.E07-08-0779.

646 Broach, J. R. (2012) 'Nutritional control of growth and development in yeast', Genetics, 192(1), pp.

647 73-105. doi: 10.1534/genetics.111.135731.

648 Cai, L. and Tu, B. P. (2012) 'Driving the Cell Cycle Through Metabolism', Annual Review of Cell and

649 Developmental Biology. doi: 10.1146/annurev-cellbio-092910-154010.

650 Carja, O. et al. (2017) 'riboviz: analysis and visualization of ribosome profiling datasets', BMC

651 bioinformatics. BioMed Central, 18(1), p. 461. doi: 10.1186/s12859-017-1873-8.

652 Chen, Z. et al. (2007) 'Restriction of DNA replication to the reductive phase of the metabolic cycle 653 protects genome integrity', Science. doi: 10.1126/science.1140958.

654 Choi, J. et al. (2017) 'A systematic genetic screen for genes involved in sensing inorganic phosphate 
655 availability in Saccharomyces cerevisiae', PLOS ONE. doi: 10.1371/journal.pone.0176085.

656 Chou, H. J. et al. (2017) 'Transcriptome-wide Analysis of Roles for tRNA Modifications in

657 Translational Regulation', Molecular Cell. doi: 10.1016/j.molcel.2017.11.002.

658 Davis, C. A. and Ares, M. (2006) 'Accumulation of unstable promoter-associated transcripts upon loss

659 of the nuclear exosome subunit Rrp6p in Saccharomyces cerevisiae', Proceedings of the National

660 Academy of Sciences. doi: 10.1073/pnas.0507783103.

661 Van Dijken, J. P. et al. (2000) 'An interlaboratory comparison of physiological and genetic properties

662 of four Saccharomyces cerevisiae strains', in Enzyme and Microbial Technology. doi: 10.1016/S0141-

663 0229(00)00162-9.

664 Fichtner, L. et al. (2003) 'Elongator's toxin-target (TOT) function is nuclear localization sequence

665 dependent and suppressed by post-translational modification', Molecular Microbiology. doi:

666 10.1046/j.1365-2958.2003.03632.x.

667 Förster, J. et al. (2003) 'Genome-scale reconstruction of the Saccharomyces cerevisiae metabolic

668 network', Genome Research. doi: 10.1101/gr.234503.

669 Goehring, A. S. (2003) ‘Urmylation: A Ubiquitin-like Pathway that Functions during Invasive Growth

670 and Budding in Yeast', Molecular Biology of the Cell. doi: 10.1091/mbc.E03-02-0079.

671 Goehring, A. S., Rivers, D. M. and Sprague, G. F. (2003) 'Attachment of the ubiquitin-related protein

672 Urm1p to the antioxidant protein Ahp1p', Eukaryotic Cell. doi: 10.1128/EC.2.5.930-936.2003.

673 González, A. and Hall, M. N. (2017) 'Nutrient sensing and TOR signaling in yeast and mammals', The

674 EMBO Journal. doi: 10.15252/embj.201696010.

675 Gresham, D. et al. (2011) 'System-level analysis of genes and functions affecting survival during

676 nutrient starvation in Saccharomyces cerevisiae', Genetics. doi: 10.1534/genetics.110.120766.

677 Gurvich, Y., Leshkowitz, D. and Barkai, N. (2017) ‘Dual role of starvation signaling in promoting

678 growth and recovery', PLoS Biology. doi: 10.1371/journal.pbio.2002039.

679 Gustilo, E. M., Vendeix, F. A. and Agris, P. F. (2008) 'tRNA's modifications bring order to gene

680 expression', Current Opinion in Microbiology. doi: 10.1016/j.mib.2008.02.003. 
681 Han, L. et al. (2018) 'Lack of 2'-O-methylation in the tRNA anticodon loop of two phylogenetically

682 distant yeast species activates the general amino acid control pathway', PLoS Genetics. doi:

683 10.1371/journal.pgen.1007288.

684 van Heerden, J. H. et al. (2014) 'Fatal attraction in glycolysis : how Saccharomyces cerevisiae

685 manages sudden transitions to high glucose', Microbial Cell. doi: 10.15698/mic2014.01.133.

686 Van Heerden, J. H. et al. (2014) 'Lost in transition: Start-up of glycolysis yields subpopulations of

687 nongrowing cells', Science. doi: 10.1126/science.1245114.

688 Hinnebusch, A. G. (1984) 'Evidence for translational regulation of the activator of general amino acid

689 control in yeast', PNAS. doi: 10.1152/japplphysiol.01106.2010.

690 Hinnebusch, A. G. (2005) 'TRANSLATIONAL REGULATION OF GCN4 AND THE GENERAL AMINO ACID

691 CONTROL OF YEAST', Annual Review of Microbiology. doi:

692 10.1146/annurev.micro.59.031805.133833.

693 Ingolia, N. T. et al. (2009) 'Genome-wide analysis in vivo of translation with nucleotide resolution

694 using ribosome profiling', Science. doi: 10.1126/science.1168978.

695 Jeong, H. et al. (2000) 'The large-scale organization of metabolic networks', Nature. doi:

$69610.1038 / 35036627$.

697 Jorgensen, P. et al. (2004) 'Picrender.Fcgi-2.Pdf', (Nasmyth 1996), pp. 2491-2505. doi:

698 10.1101/gad.1228804.).

699 Kim, D., Langmead, B. and Salzberg, S. L. (2015) 'HISAT: a fast spliced aligner with low memory

700 requirements', Nature methods. 2015/03/09, 12(4), pp. 357-360. doi: 10.1038/nmeth.3317.

701 Klassen, R. et al. (2016) 'TRNA anticodon loop modifications ensure protein homeostasis and cell

702 morphogenesis in yeast', Nucleic Acids Research. doi: 10.1093/nar/gkw705.

703 Klosinska, M. M. et al. (2011) 'Yeast cells can access distinct quiescent states', Genes and

704 Development. doi: 10.1101/gad.2011311.

705 Kwapisz, M. et al. (2008) 'Mutations of RNA polymerase II activate key genes of the nucleoside

706 triphosphate biosynthetic pathways', EMBO Journal. doi: 10.1038/emboj.2008.165. 
707 Laxman, S. et al. (2013) 'Sulfur Amino Acids Regulate Translational Capacity and Metabolic

708 Homeostasis through Modulation of tRNA Thiolation', Cell, 154(2), pp. 416-429. doi:

709 10.1016/j.cell.2013.06.043.

710 Laxman, S. and Tu, B. P. (2011) 'Multiple TORC1-associated proteins regulate nitrogen starvation-

711 dependent cellular differentiation in Saccharomyces cerevisiae', PLOS ONE. doi:

$71210.1371 /$ journal.pone.0026081.

713 Leidel, S. et al. (2009) 'Ubiquitin-related modifier Urm1 acts as a sulphur carrier in thiolation of

714 eukaryotic transfer RNA', Nature. doi: 10.1038/nature07643.

715 Lemire, J. M. et al. (1985) 'Regulation of repressible acid phosphatase gene transcription in

716 Saccharomyces Regulation of Repressible Acid Phosphatase Gene Transcription in Saccharomyces

717 cerevisiae', Molecular and cellular biology. doi: 10.1128/MCB.5.8.2131.Updated.

718 Lenburg, M. E. and O’Shea, E. K. (1996) 'Signaling phosphate starvation', Trends in Biochemical

719 Sciences. doi: 10.1016/0968-0004(96)10048-7.

720 Levy, S. et al. (2011) 'The competitive advantage of a dual-transporter system', Science. doi:

$721 \quad 10.1126 /$ science.1207154.

722 Li, W. et al. (2017) 'Riborex: fast and flexible identification of differential translation from Ribo-seq

723 data', Bioinformatics (Oxford, England). 2017/01/31. Oxford University Press, 33(11), pp. 1735-1737.

724 doi: 10.1093/bioinformatics/btx047.

725 Lillie, S. H. and Pringle, J. R. (1980) ‘Reserve carbohydrate metabolism in Saccharomyces cerevisiae:

726 responses to nutrient limitation', Journal of Bacteriology.

727 Liu, N.-N. et al. (2017) 'Phosphate is the third nutrient monitored by TOR in Candida albicans and

728 provides a target for fungal-specific indirect TOR inhibition', Proceedings of the National Academy of

729 Sciences. doi: 10.1073/pnas.1617799114.

730 Ljungdahl, P. O. and Daignan-Fornier, B. (2012) 'Regulation of amino acid, nucleotide, and phosphate

731 metabolism in Saccharomyces cerevisiae', Genetics, 190(3), pp. 885-929. doi:

$732 \quad 10.1534 /$ genetics.111.133306. 
733 Love, M. I., Huber, W. and Anders, S. (2014) 'Moderated estimation of fold change and dispersion for

734 RNA-seq data with DESeq2', Genome Biology, 15(12), p. 550. doi: 10.1186/s13059-014-0550-8.

735 Madden, S., Johnson, D. and Bergman, L. (1990) 'Molecular and expression analysis of the negative

736 regulators involved in the transcriptional regulation of acid phosphatase production in

737 Saccharomyces cerevisiae.', Molecular and cellular ..., 10(11), pp. 5950-5957. doi:

$738 \quad$ 10.1128/MCB.10.11.5950.

739 Madden, S. L. et al. (1988) 'negative trans-acting', 16(6), pp. 2625-2637.

740 Martin, M. (2011) 'Cutadapt removes adapter sequences from high-throughput sequencing reads',

741 EMBnet.journal; Vol 17, No 1: Next Generation Sequencing Data Analysis. doi: 10.14806/ej.17.1.200.

742 Mason, P. W. et al. (1981) 'The importance of inorganic phosphate in regulation of energy

743 metabolism of Streptococcus lactis', Journal of Biological Chemistry.

744 McGlincy, N. J. and Ingolia, N. T. (2017) 'Transcriptome-wide measurement of translation by

745 ribosome profiling', Methods. doi: 10.1016/j.ymeth.2017.05.028.

746 McMahon, M. and Ruggero, D. (2018) 'A wobbly road to drug resistance in melanoma: tRNA-

747 modifying enzymes in translation reprogramming', The EMBO Journal. doi:

$748 \quad 10.15252 /$ embj.201899978.

749 McNaughton, R. L. et al. (2010) 'Probing in vivo Mn2+ speciation and oxidative stress resistance in

750 yeast cells with electron-nuclear double resonance spectroscopy', Proceedings of the National

751 Academy of Sciences. doi: 10.1073/pnas.1009648107.

752 Nakai, Y., Nakai, M. and Hayashi, H. (2008) 'Thio-modification of yeast cytosolic tRNA requires a

753 ubiquitin-related system that resembles bacterial sulfur transfer systems', Journal of Biological

754 Chemistry. doi: 10.1074/jbc.M804043200.

755 Nedialkova, D. D. and Leidel, S. A. (2015) 'Optimization of Codon Translation Rates via tRNA

756 Modifications Maintains Proteome Integrity', Cell. doi: 10.1016/j.cell.2015.05.022.

757 Noma, A., Sakaguchi, Y. and Suzuki, T. (2009) 'Mechanistic characterization of the sulfur-relay system

758 for eukaryotic 2-thiouridine biogenesis at tRNA wobble positions', Nucleic Acids Research. doi: 
10.1093/nar/gkn1023.

760 Ogawa, N. et al. (1995) 'Functional domains of Pho81p, an inhibitor of Pho85p protein kinase, in the

761 transduction pathway of Pi signals in Saccharomyces cerevisiae.', ... and cellular biology. doi:

$762 \quad 10.1091 / \mathrm{mbc} .11 .12 .4309$.

763 Ogawa, N., DeRisi, J. and Brown, P. O. (2000) 'New Components of a System for Phosphate

764 Accumulation and Polyphosphate Metabolism in Saccharomyces cerevisiae Revealed by Genomic

765 Expression Analysis', Molecular Biology of the Cell. doi: 10.1091/mbc.11.12.4309.

766 Phizicky, E. M. and Hopper, A. K. (2010) 'tRNA biology charges to the front', Genes and Development.

767 doi: 10.1101/gad.1956510.

768 Rapino, F. et al. (2018) 'Codon-specific translation reprogramming promotes resistance to targeted

769 therapy', Nature. doi: 10.1038/s41586-018-0243-7.

770 Rezgui, V. A. N. et al. (2013) 'tRNA tKUUU, tQUUG, and tEUUC wobble position modifications fine-

771 tune protein translation by promoting ribosome A-site binding', Proceedings of the National

772 Academy of Sciences. doi: 10.1073/pnas.1300781110.

773 Rolfes, R. J. and Hinnebusch, A. G. (1993) 'Translation of the yeast transcriptional activator GCN4 is 774 stimulated by purine limitation: implications for activation of the protein kinase GCN2.', Molecular 775 and Cellular Biology. doi: 10.1128/MCB.13.8.5099.Updated.

776 Saldanha, A., Brauer, M. and Botstein, D. (2004) 'Nutritional Homeostasis in Batch and Steady-State

777 Culture of Yeast', Molecular biology of the cell. doi: 10.1091/mbc.E04-04-0306.

778 Scheidt, V. et al. (2014) 'Loss of wobble uridine modification in tRNA anticodons interferes with TOR

779 pathway signaling', Microbial Cell. doi: 10.15698/mic2014.12.179.

780 Schmitz, J. et al. (2008) 'The sulfurtransferase activity of Uba4 presents a link between ubiquitin-like

781 protein conjugation and activation of sulfur carrier proteins', Biochemistry. doi: 10.1021/bi800477u.

782 Schneider, K. R., Smith, R. L. and O’Shea, E. K. (1994) 'Phosphate-regulated inactivation of the

783 kinase? PHO80-PHO85 by the CDK inhibitor PHO81', Science. doi: 10.1126/science.7939631.

784 Secco, D. et al. (2012) 'Phosphate homeostasis in the yeast Saccharomyces cerevisiae, the key role of 
785 the SPX domain-containing proteins', FEBS Letters. doi: 10.1016/j.febslet.2012.01.036.

786 Shi, L. et al. (2010) 'Trehalose Is a Key Determinant of the Quiescent Metabolic State That Fuels Cell

787 Cycle Progression upon Return to Growth', Molecular biology of the cell. doi: 10.1091/mbc.E10.

788 Shi, L. and Tu, B. P. (2013) 'Acetyl-CoA induces transcription of the key G1 cyclin CLN3 to promote

789 entry into the cell division cycle in Saccharomyces cerevisiae', Proceedings of the National Academy

790 of Sciences. doi: 10.1073/pnas.1302490110.

791 Slavov, N. and Botstein, D. (2011) ‘Coupling among growth rate response, metabolic cycle, and cell

792 division cycle in yeast', Molecular Biology of the Cell. doi: 10.1091/mbc.E11-02-0132.

793 Subtelny, A. O. et al. (2014) 'Poly(A)-tail profiling reveals an embryonic switch in translational

794 control', Nature. 2014/01/29, 508(7494), pp. 66-71. doi: 10.1038/nature13007.

795 Thomas, D. and Surdin-Kerjan, Y. (1997) 'Metabolism of sulfur amino acids in Saccharomyces

796 cerevisiae', Microbiology and molecular biology reviews : MMBR. doi: 10.1186/1471-2148-8-39.

797 Toh-E, A. and Shimauchi, T. (1986) 'Cloning and sequencing of the PHO80 gene and CEN15 of

798 Saccharomyces cerevisiae', Yeast. doi: 10.1002/yea.320020209.

799 Tu, B. P. et al. (2005) 'Logic of the Yeast Metabolic Cycle : of Cellular Processes', Science (New York, $800 \quad$ N.Y.). doi: 10.1126/science.1120499.

801 Virgilio, C. D. E. et al. (1993) 'Disruption of TPS2, the gene encoding the 100-kDa subunit of the

802 trehalose-6-phosphate synthase / phosphatase complex in Saccharomyces cerevisiae, causes

803 accumulation of trehalose-6-phosphate and loss of trehalose-6-phosphate phosphatase activity',

804 Eur. J. Biochem., 212, pp. 315-323.

805 Walvekar, A., Rashida, Z., et al. (2018) 'A versatile LC-MS/MS approach for comprehensive,

806 quantitative analysis of central metabolic pathways', Wellcome Open Research, 3(0), p. 122. doi:

807 10.12688/wellcomeopenres.14832.1.

808 Walvekar, A., Srinivasan, R., et al. (2018) 'Methionine coordinates a hierarchically organized anabolic

809 program enabling proliferation.', bioRxiv. doi: https://doi.org/10.1101/249367.

810 Warner, J. R. (2001) 'Nascent ribosomes', Cell. doi: 10.1016/S0092-8674(01)00531-1. 
811 Weinberg, D. E. et al. (2016) 'Improved Ribosome-Footprint and mRNA Measurements Provide

812 Insights into Dynamics and Regulation of Yeast Translation', Cell Reports. doi:

813 10.1016/j.celrep.2016.01.043.

814 Wilson, R. A. et al. (2007) 'Tps1 regulates the pentose phosphate pathway, nitrogen metabolism and

815 fungal virulence', The EMBO journal. 2007/07/19. Nature Publishing Group, 26(15), pp. 3673-3685.

816 doi: 10.1038/sj.emboj.7601795.

817 Wullschleger, S., Loewith, R. and Hall, M. N. (2006) 'TOR signaling in growth and metabolism', Cell.

818 doi: 10.1016/j.cell.2006.01.016.

819 Wykoff, D. D. and O’Shea, E. K. (2001) 'Phosphate transport and sensing in Saccharomyces

820 cerevisiae', Genetics. doi: 10.1534/genetics.166.4.1727.

821 Yarian, C. et al. (2002) 'Accurate translation of the genetic code depends on tRNA modified

822 nucleosides', Journal of Biological Chemistry. doi: 10.1074/jbc.M200253200.

823 Yoshida, K. et al. (1989) 'Mode of expression of the positive regulatory genes PHO2 and PHO4 of the

824 phosphatase regulon in Saccharomyces cerevisiae', MGG Molecular \& General Genetics. doi:

825 10.1007/BF00330939.

826 Zaman, S. et al. (2008) 'How Saccharomyces Responds to Nutrients', Annual Review of Genetics. doi:

827 10.1146/annurev.genet.41.110306.130206.

828 Zinshteyn, B. and Gilbert, W. V (2013) 'Loss of a conserved tRNA anticodon modification perturbs

829 cellular signaling. TL - 9', PLoS genetics. doi: 10.1371/journal.pgen.1003675. 
A

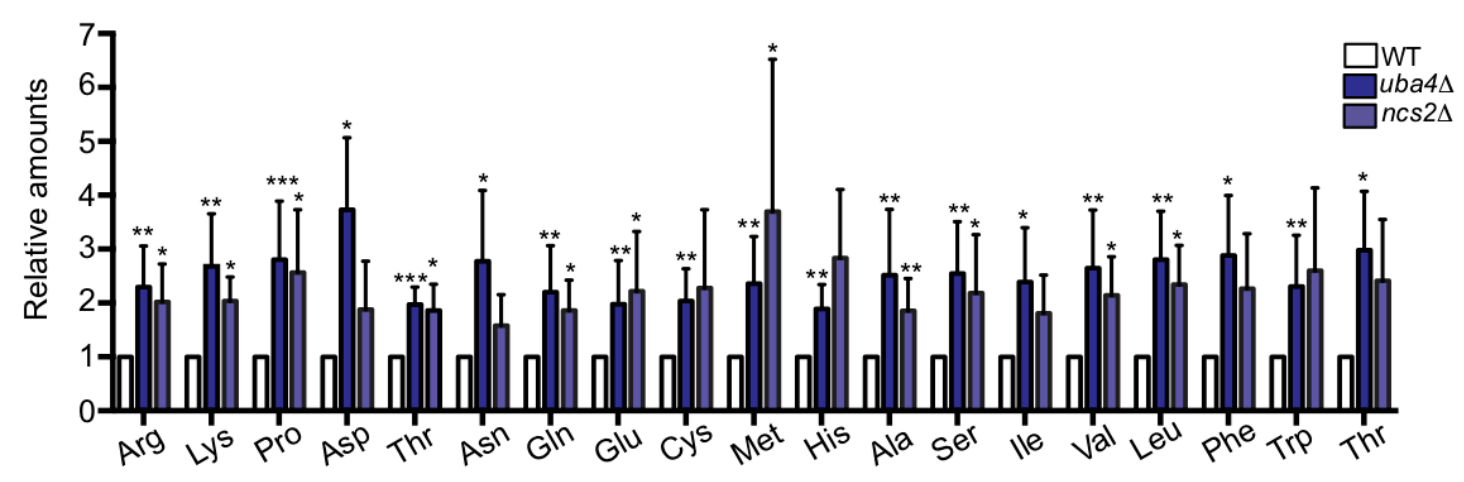

B

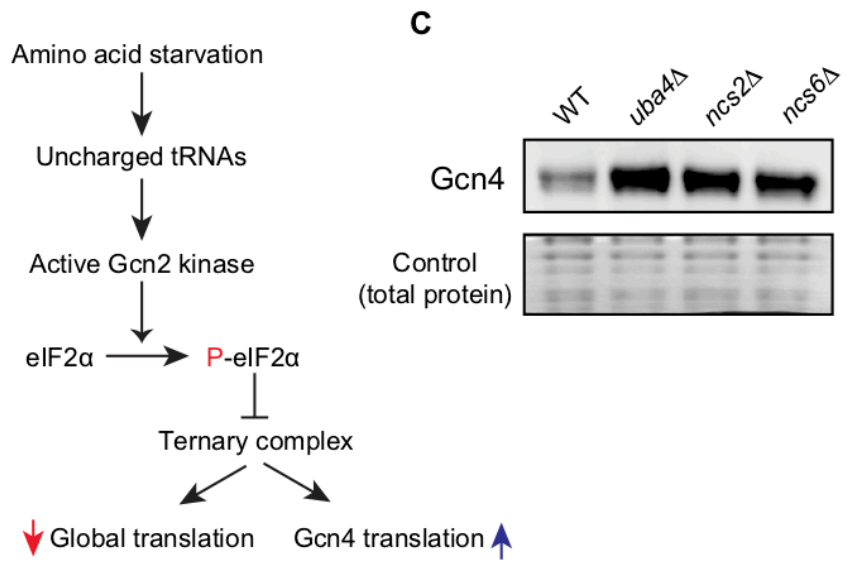

E
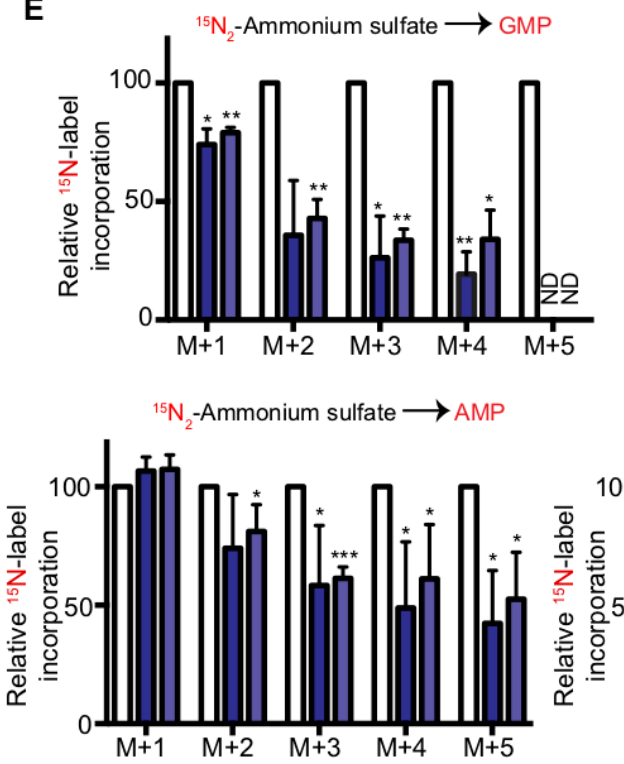

D

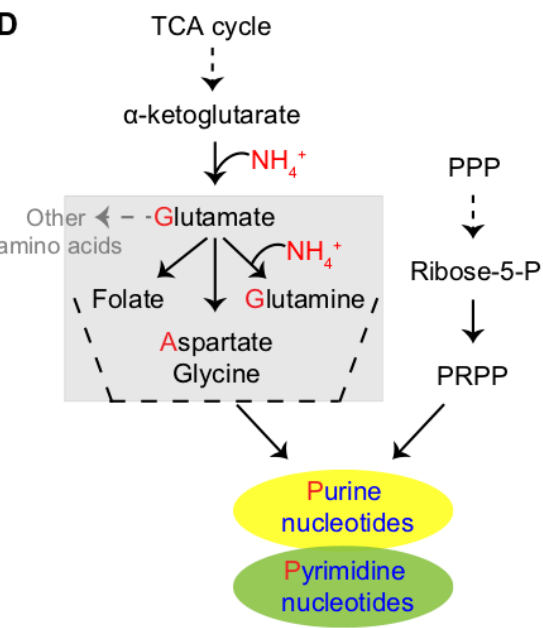

$\mathbf{F}$

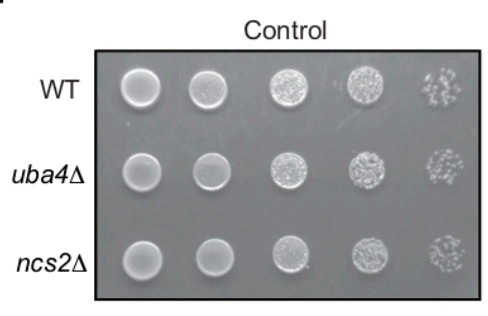

8-Aza adenine

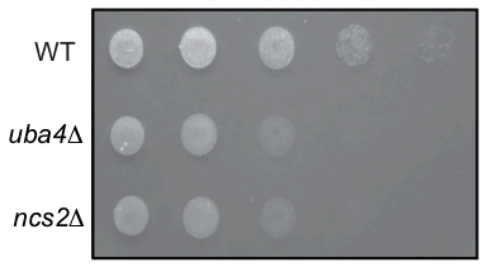

Figure 1 
834 (A) Intracellular pools of amino acids are increased in tRNA thiolation mutants. Steady-state amino 835 acid amounts were measured in wild-type (WT) and tRNA thiolation mutant cells (uba4 $\Delta$ and $n c s 2 \Delta$ ) 836 grown in minimal media by targeted liquid chromatography/mass spectrometry (LC-MS/MS). Data are 837 displayed as means \pm SD, $n>=3 .{ }^{*} p<0.05,{ }^{* *} p<0.01,{ }^{* * *} p<0.001$.

838 (B) A schematic representation illustrating the induction of Gcn4 translational upon amino acid starvation, as mediated by the Gcn2 kinase, and phosphorylation of the elF2 $\alpha$ initiation factor.

840 (C) Gcn4 protein is increased in tRNA thiolation mutants. Western blots indicating Gcn4 protein levels

841 (Gcn4 tagged with HA epitope at the endogenous locus) in WT and tRNA thiolation mutant cells 842 (uba4 $\Delta, n c s 2 \Delta$ and $n c s 6 \Delta$ ) grown in minimal media, as detected using an anti-HA antibody. A 843 representative blot obtained from three biological replicates $(n=3)$ is shown. Also see figures $S 1 A$, 844 S1B and S1C.

845 (D) A schematic representation of de novo nucleotide (purine and pyrimidine) biosynthesis as made 846 from its precursors- amino acids, folates and PRPP (5-Phopsphoribosyl-1-Pyrophosphate).

847 (E) Nucleotide synthesis is decreased in tRNA thiolation mutants. WT and tRNA thiolation mutant 848 cells (uba4 $\Delta$ and ncs $2 \Delta$ ) grown in minimal media were pulse-labelled with ${ }^{15} \mathrm{~N}_{2}$-labelled ammonium 849 sulfate for 90 minutes to measure newly synthesized nucleotides (GMP, AMP and CMP) using 850 targeted LC-MS/MS. Label incorporation in tRNA thiolation mutant cells relative to WT is plotted, 851 where label incorporation in WT was set to $100 \%$. The incorporation of ${ }^{15} \mathrm{~N}$ atoms from ${ }^{15} \mathrm{~N}_{2}$-labelled 852 ammonium sulfate into nucleotides is represented as $M+n$, where $n$ is the number of ${ }^{15} \mathrm{~N}$-labelled 853 atoms. Data are displayed as means $\pm S D, n=3$ for AMP and CMP, $n=2$ for GMP. ${ }^{*} p<0.05,{ }^{* *} p<0.01$, $854{ }^{* * *} p<0.001$. Also see Figures S2A, S2B and S2D.

855 (F) tRNA thiolation mutants exhibit increased sensitivity to 8-azaadenine. WT and tRNA thiolation 856 mutant cells ( $u b a 4 \Delta$ and $n c s 2 \Delta$ ) grown in minimal media were spotted on minimal media agar plates 857 containing $300 \mu \mathrm{g} / \mathrm{ml}$ 8-Aza adenine. Also see Figures S2C. 
A

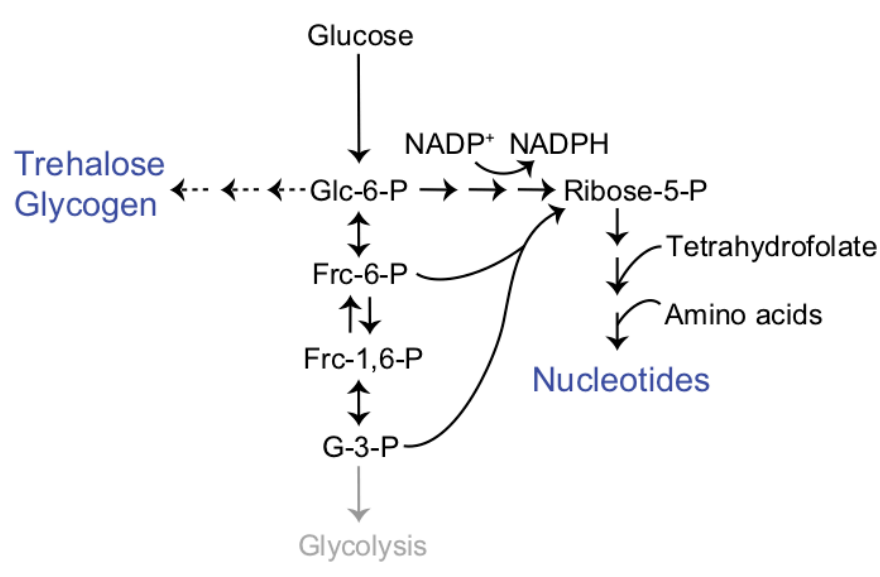

B
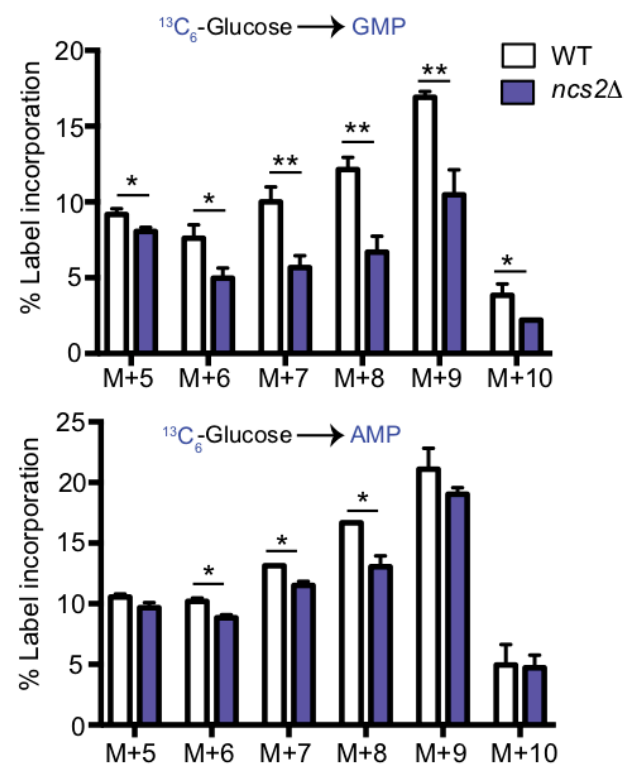

C

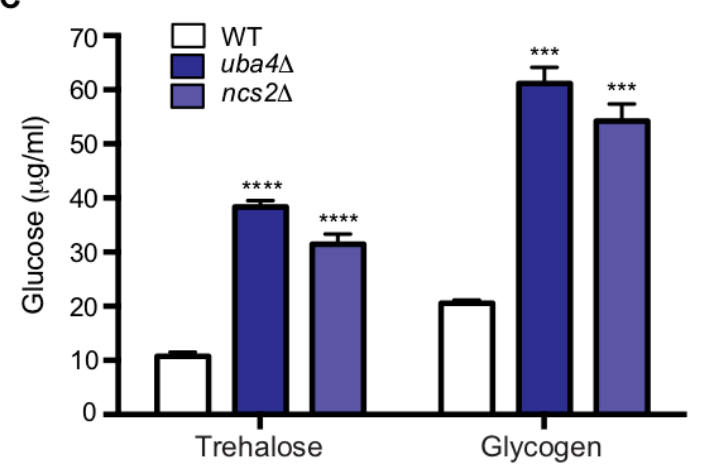

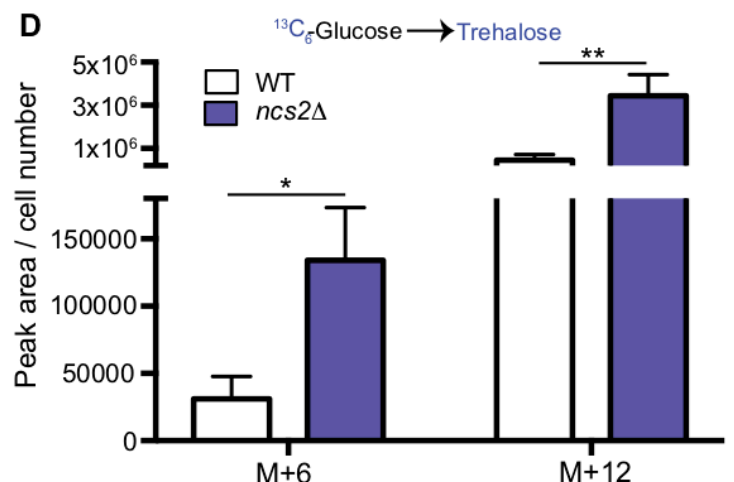

Figure 2: Carbon flux is routed towards storage carbohydrates in thiolation mutants

(A) Schematic representation depicting nucleotide and storage carbohydrates (trehalose and glycogen) biosynthesis, starting from a common precursor, glucose-6-phosphate. In normal conditions, flux is typically higher towards the pentose phosphate pathway and eventually nucleotides.

(B) Nucleotide synthesis is decreased in tRNA thiolation mutants. WT and tRNA thiolation mutant cells (ncs2 $\Delta$ ) grown in minimal media were pulse-labelled with $\left[\mathrm{U}-{ }^{13} \mathrm{C}_{6}\right]$-labelled glucose for 10 minutes to measure newly synthesized nucleotides (GMP and AMP) using mass spectrometry. Percent label incorporation in WT and tRNA thiolation mutant cells was plotted. The incorporation of ${ }^{13} \mathrm{C}$ atoms from $\left[\mathrm{U}^{13} \mathrm{C}_{6}\right]$-labelled glucose into nucleotides is represented as $M+n$, where $n$ is the number of ${ }^{13} \mathrm{C}$-labelled atoms (with all five carbons labelled in the ribose sugar). Data are displayed as means $\pm S D, n=3$ for GMP and $n=2$ for AMP. ${ }^{*} p<0.05$, ${ }^{* *} p<0.01$. Also see Figures $S 3 A$ and S3B.

(C) Steady-state trehalose and glycogen amounts are increased in tRNA thiolation mutants. Trehalose and glycogen content of WT and tRNA thiolation mutant cells ( $u b a 4 \Delta$ and ncs $2 \Delta$ ) grown in minimal media was plotted. Data are displayed as means $\pm S D, n=4$ biological replicates with two technical replicates each for trehalose, and $n=3$ biological replicates with two technical replicates each for glycogen. ${ }^{* * *} p<0.001,{ }^{* * *} p<0.0001$. Also see Figures S3C.

(D) Trehalose synthesis is increased in tRNA thiolation mutant. WT and tRNA thiolation mutant cells (ncs $2 \Delta$ ) grown in minimal media were pulse-labelled with $\left[\mathrm{U}-{ }^{13} \mathrm{C}_{6}\right]$-labelled glucose for 5 minutes to measure newly synthesized trehalose using targeted LC-MS/MS. Normalized peak area of trehalose in WT and tRNA thiolation mutant cells was plotted. The incorporation of ${ }^{13} \mathrm{C}$ atoms from $\left[\mathrm{U}-{ }^{13} \mathrm{C}_{6}\right]-$ labelled glucose into trehalose is represented as $M+n$, where $n$ is the number of ${ }^{13} \mathrm{C}$-labelled atoms. Data are displayed as means $\pm S D, n=3 .{ }^{*} p<0.05,{ }^{* *} p<0.01$. 
A
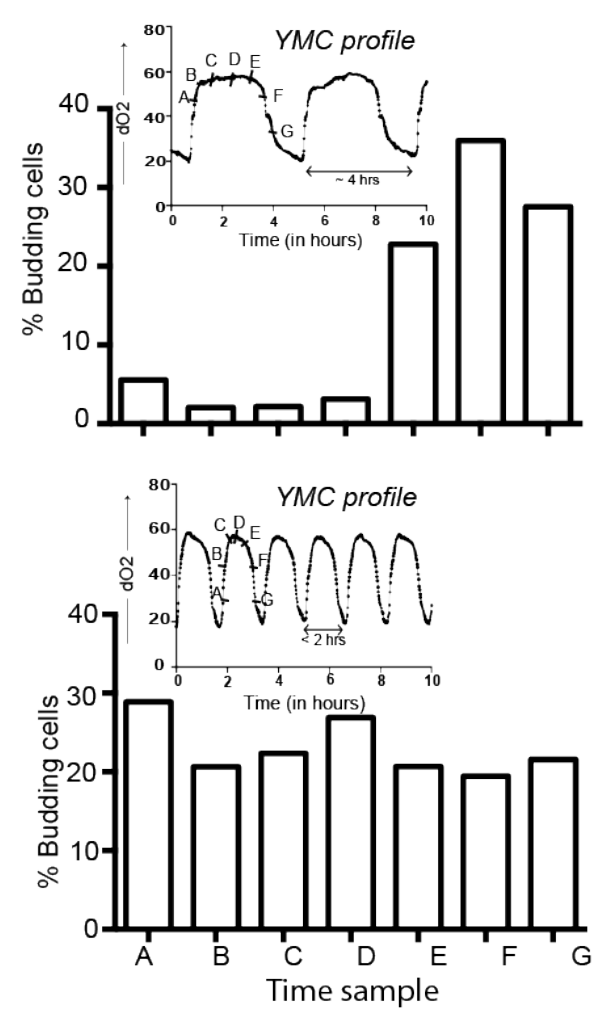

C

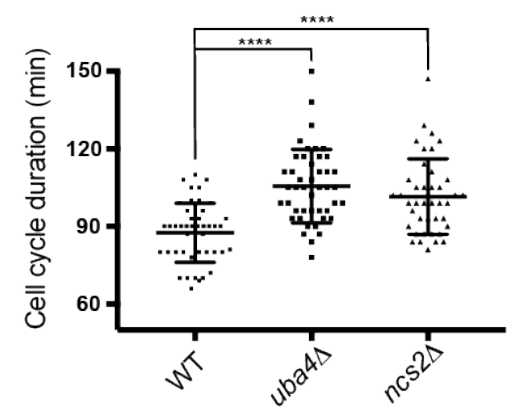

D

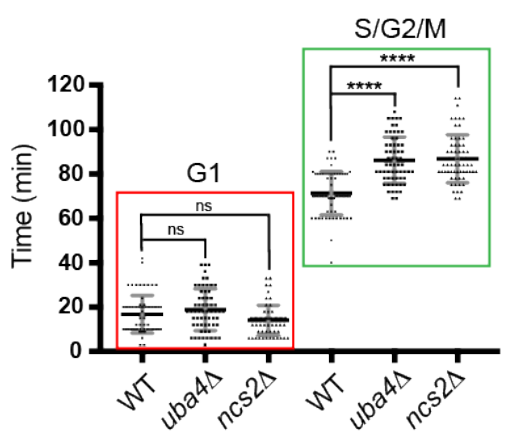

B

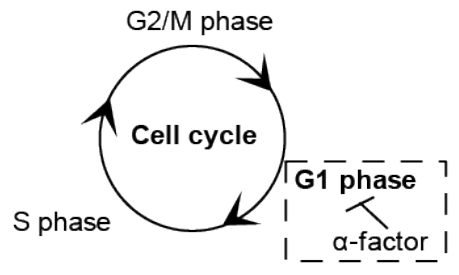

WT

$u b a 4 \Delta$

E

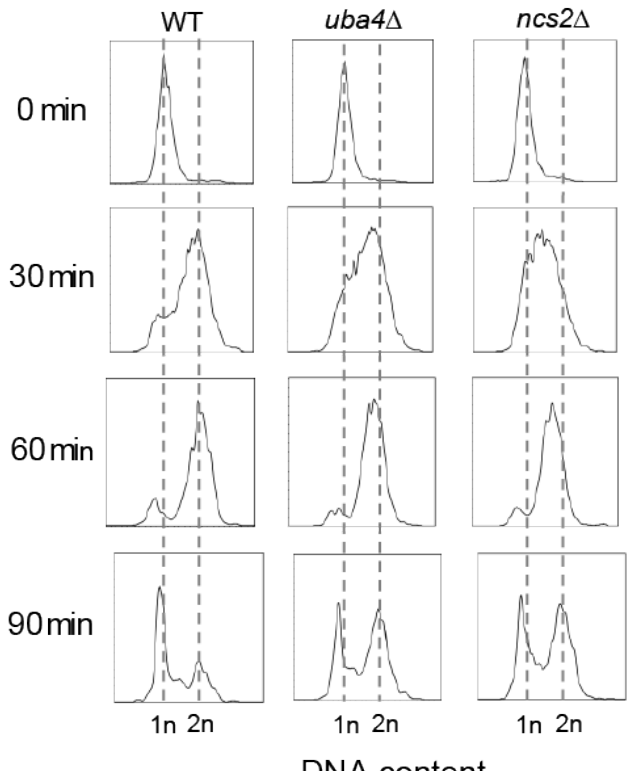

DNA content

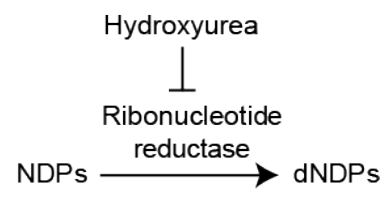

$+\mathrm{HU}$
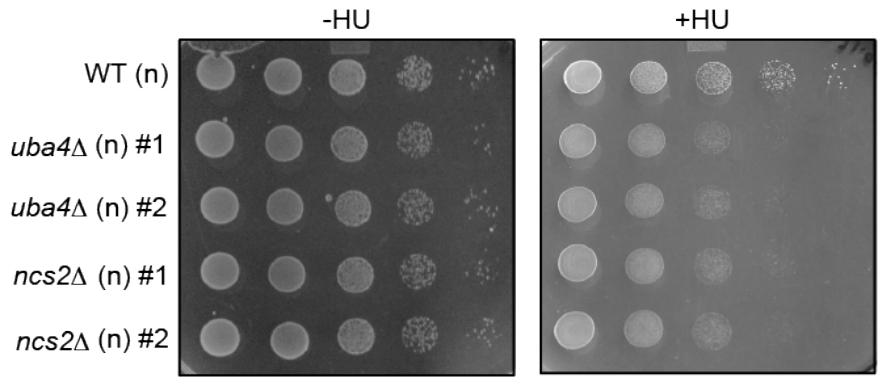

Figure 3

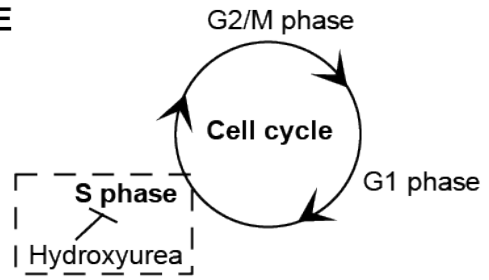

$-\mathrm{HU}$
Figure 3 tRNA thiolation couples cellular metabolic state with normal cell cycle progression

884 (A) tRNA thiolation mutants exhibit asynchronous cell division and disrupted yeast metabolic cycles. WT and tRNA thiolation mutant cells ( $u b a 4 \Delta$ ) growing in chemostat cultures under conditions of normal yeast metabolic cycles, were sampled at 20 minute time intervals. Percentage of budded cells represents the fraction of cells in S/G2/M phases of the cell cycle. At least 200 cells were analysed for each time point, for the respective strains. Inset: the oxygen consumption profiles of the WT and tRNA thiolation mutant metabolic cycles are represented. Note that the metabolic cycles of thiolation mutants are disrupted. 
891 (B) tRNA thiolation mutants show delayed cell cycle progression. The DNA content of WT and tRNA 892 thiolation mutant cells ( $u b a 4 \Delta$ and $n c s 2 \Delta$ ) grown in minimal media, during G1 arrest ( $0 \mathrm{~min}$ ) and after 893 release from $\mathrm{G} 1$-arrest $(30,60$ and $90 \mathrm{~min})$ was determined by flow cytometry analysis, and is 894 presented.

895 (C and D) Cell cycle duration, distribution of $\mathrm{G} 1$ phase duration (red) and S/G2/M phase durations 896 (green) for WT and tRNA thiolation mutant cells ( $u b a 4 \Delta$ and ncs $2 \Delta$ ) were measured in single cells 897 using time lapse live-cell microscopy. We determined the G1 duration (starting from the time of 898 complete division of mother and daughter cells to bud emergence), and S/G2/M durations (from the 899 time of bud emergence to complete division of mother and daughter cells) for only first generation 900 mother cells. At least 100 cells were analysed for each strain. ns denotes non-significant difference. $901{ }^{* * * *} p<0.0001$.

902 (E) tRNA thiolation mutants exhibit increased HU sensitivity. WT and tRNA thiolation mutant cells 903 (uba4 $\Delta$ and $n c s 2 \Delta$ ) grown in minimal media were spotted on minimal media agar plates containing $904150 \mathrm{mM} \mathrm{HU}$. Also see Figures S4A and S4B. 


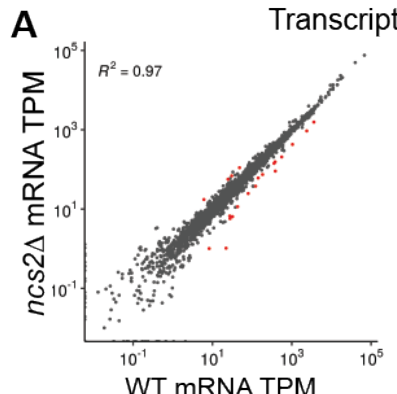

B

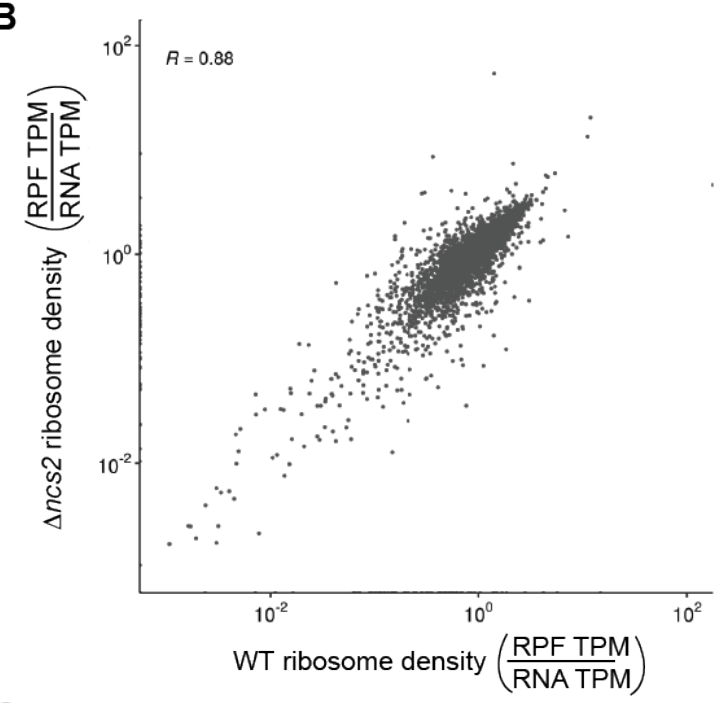

C Amino acid biosynthesis

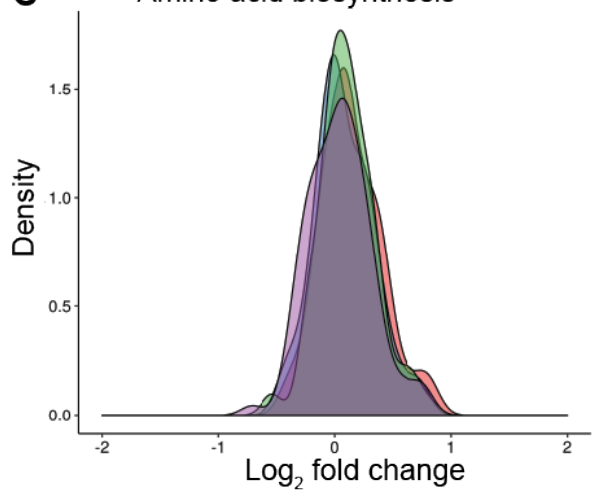

E

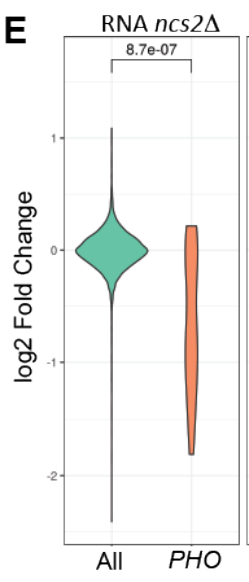

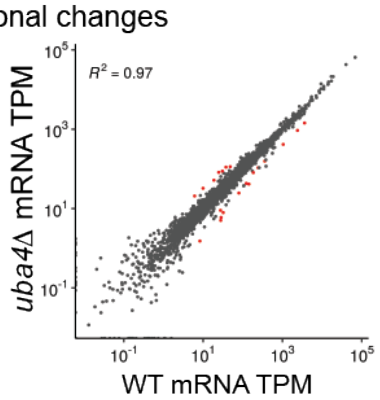

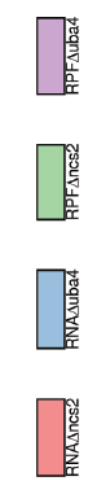

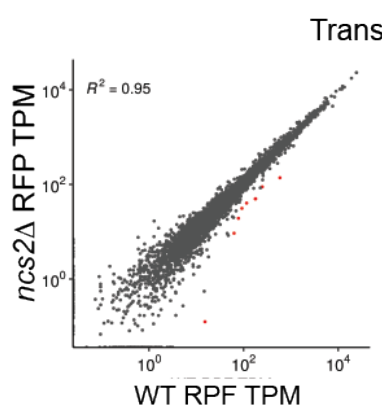
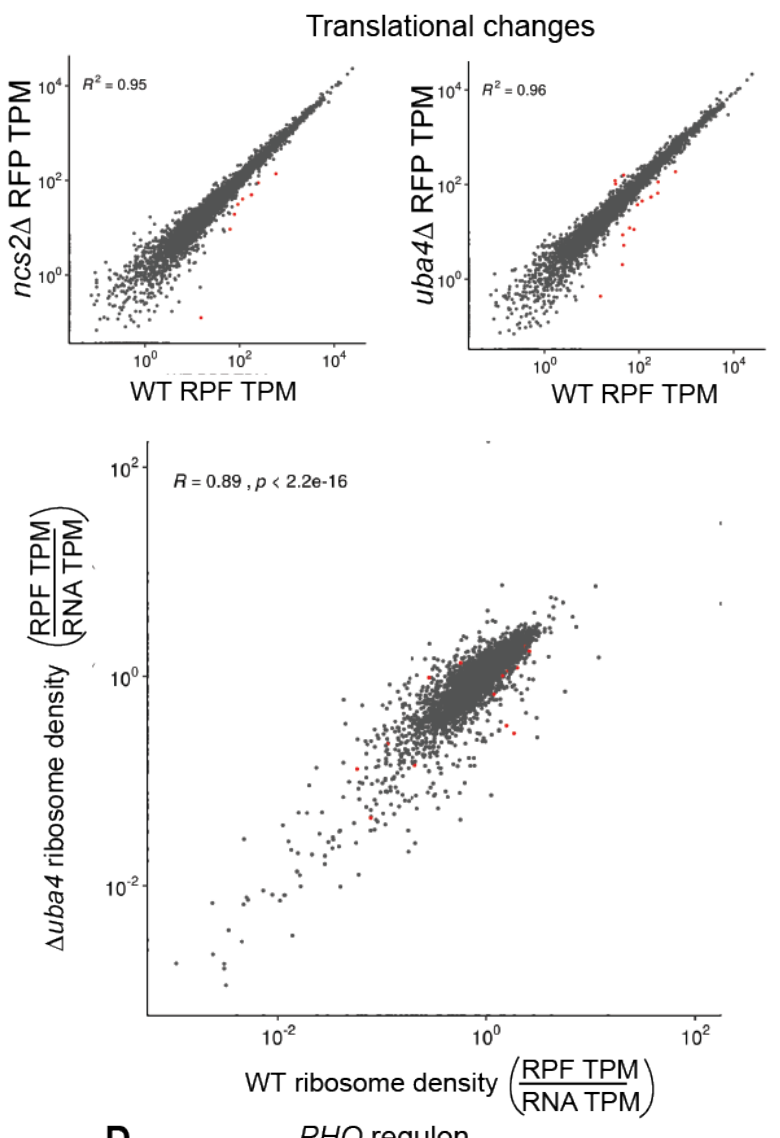

D $\quad$ PHO regulon

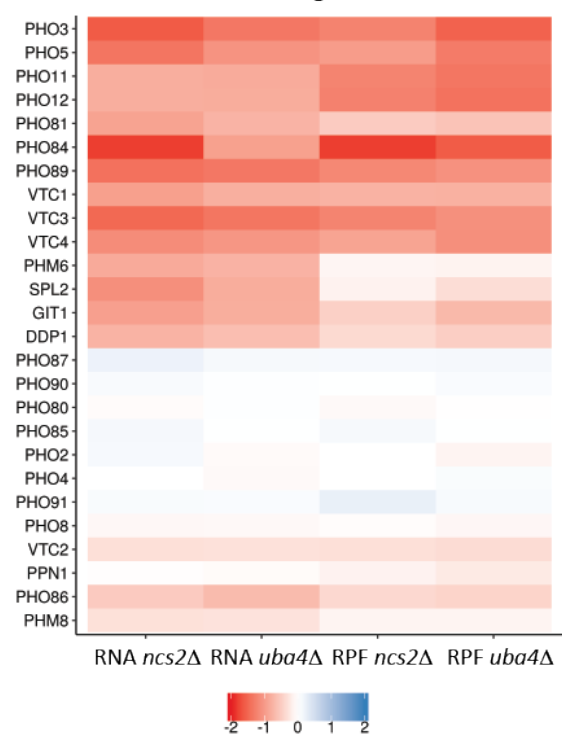

Figure 4 Loss of tRNA thiolation results in reduced phosphate homeostasis $(P H O)$ related 
909 (A) Correlation plots, for WT and tRNA thiolation mutant cells ( $n c s 2 \Delta$ and $u b a 4 \Delta$ ) for both gene 910 expression (transcript) and ribosome footprint (translation) changes is shown. The coefficients of 911 determination $\left(R^{2}\right)$ values are shown, along with their significance ( $p$ values). Note: Very few 912 differentially regulated genes were observed, and these are indicated as red points. Also see Figures 913 S5A, S5B and S5C.

914 (B) Correlation plots comparing ribosome densities (RPF TPM/RNA TPM) for the thiolation mutants 915 (ncs2 2 or $u b a 4 \Delta$ respectively) with WT cells. Note: overall ribosome densities for thiolation mutants 916 correlate exceptionally well with ribosome densities in WT cells, with the correlation coefficients (R) > 9170.88 in both comparisons.

918 (C) A density plot, representing changes in expression (transcript and ribosome footprints) of genes 919 associated with amino acid biosynthetic pathways. In general, amino acid biosynthesis genes were 920 upregulated in tRNA thiolation mutant cells ( $u b a 4 \Delta$ and $n c s 2 \Delta$ ). Also see Figures S6A, S6B and S6C.

921 (D) Heat map depicting changes in expression (transcript and ribosome footprints) of genes 922 associated with the phosphate $(P H O)$ regulon, in the tRNA thiolation mutant cells (uba4 $\Delta$ and $n c s 2 \Delta$ ), 923 compared to WT cells, at the transcript and ribosome-footprint levels (log2 2-fold changes).

924 (E) A density plot with a statistical comparison between WT and tRNA thiolation mutants, for changes 925 in transcript or ribosome footprints of all genes in the genome, or only the $P H O$ regulon. Note: $p<10^{-7}$ 926 for all the comparison datasets. Also see Figure S6D. 

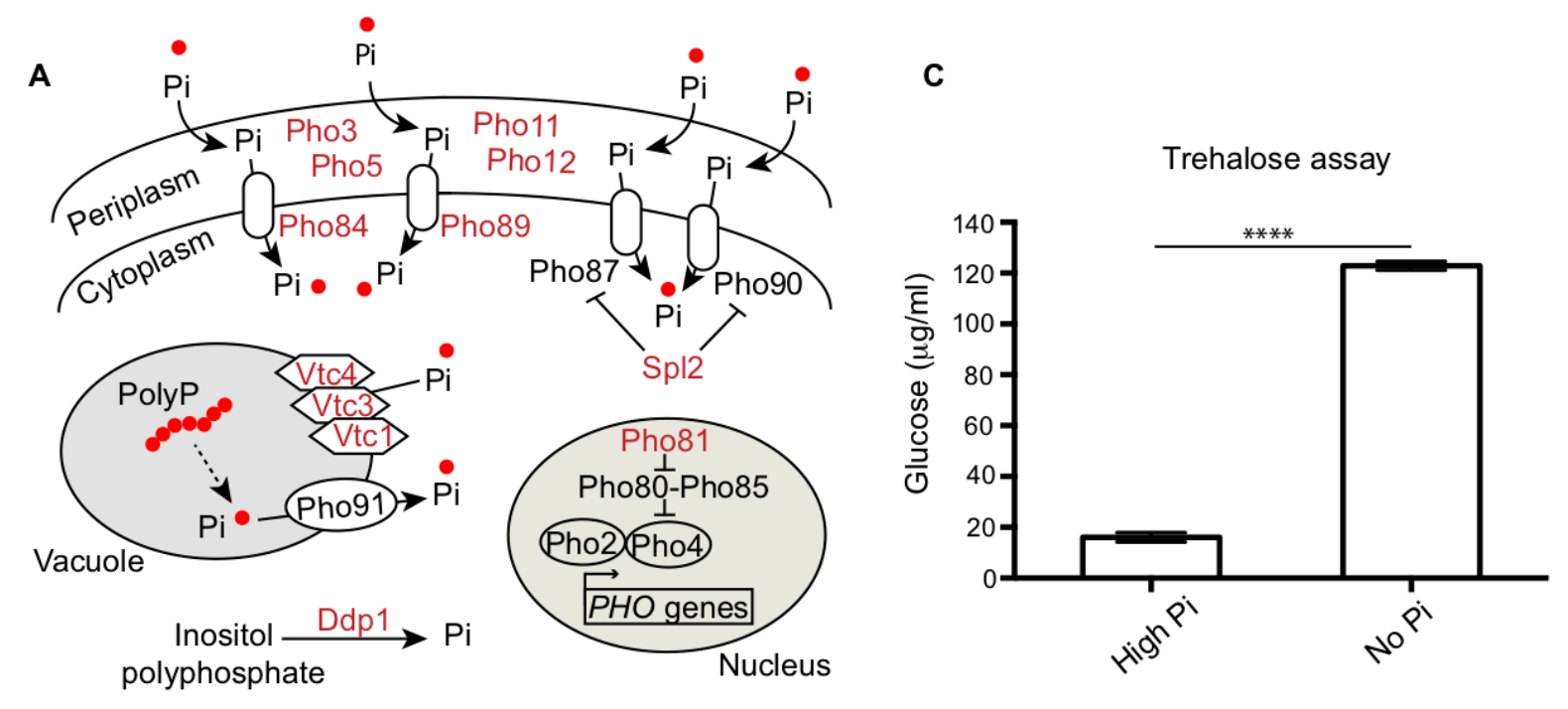

B

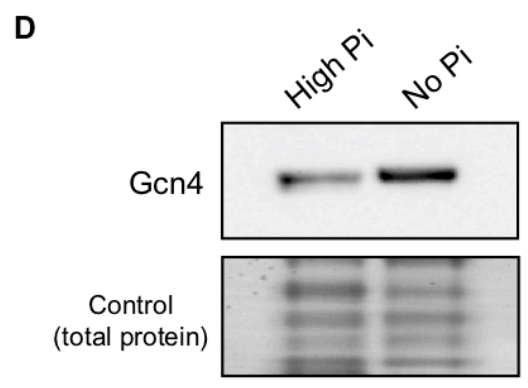

Figure 5

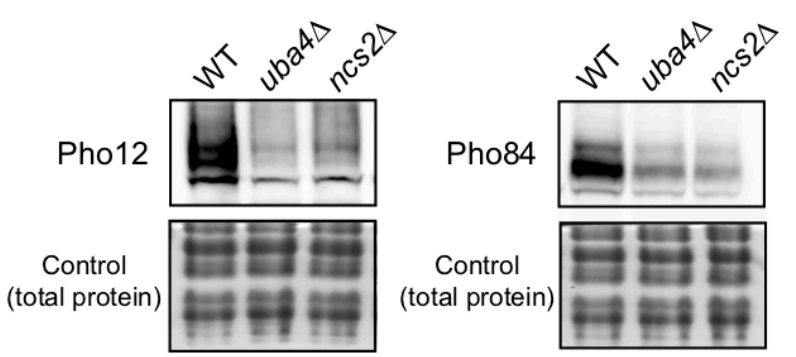

\section{Figure 5: Phosphate depletion in wild-type cells phenocopies tRNA thiolation mutants}

930 (A) A schematic representation of $\mathrm{PHO}$ regulon related genes, and their roles. Pho84 and Pho89 are high affinity phosphate transporters, Pho87 and Pho90 are low affinity phosphate transporters, Spl2 is a negative regulator of low affinity phosphate transporters, Pho3, Pho5, Pho11 and Pho12 are secreted acid phosphatases, Pho80-Pho85 is a cyclin-dependent kinase (CDK) complex, Pho81 is a CDK inhibitor, Pho2 and Pho4 are transcription factors, Vtc1, Vtc3 and Vtc4 are involved in vacuolar polyphosphate accumulation, Pho91 is a vacuolar phosphate transporter, Ddp1 and Ppn1 are polyphosphatases. Genes marked in red are down-regulated in tRNA thiolation mutant cells (uba4 $\Delta$ and $n \operatorname{cs} 2 \Delta$ ).

938 (B) Pho12 and Pho84 proteins are decreased in tRNA thiolation mutants. Pho12 and Pho84 protein levels (Pho12 and Pho84 tagged with FLAG epitope at their endogenous loci) in WT and tRNA thiolation mutant cells ( $u b a 4 \Delta, n c s 2 \Delta$ and $n c s 6 \Delta$ ) grown in minimal media were detected by Western blot analysis using an anti- FLAG antibody. A representative blot is shown $(n=3)$.

(C) Trehalose amounts are increased upon phosphate starvation. Trehalose content of WT cells grown in high and no Pi media was plotted. Data are displayed as means $\pm S D, n=3$. ${ }^{* * *} p<0.0001$.

(D) Gcn4 protein is increased upon phosphate starvation. Gcn4 protein levels (Gcn4 tagged with HA epitope at the endogenous locus) in WT grown in high and no Pi media were detected by Western blot analysis using an anti-HA antibody. A representative blot is shown $(n=3)$. 
A

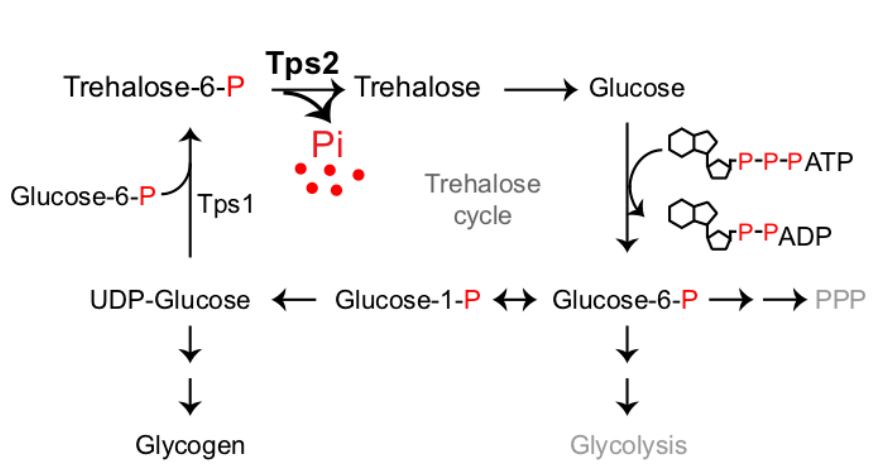

C
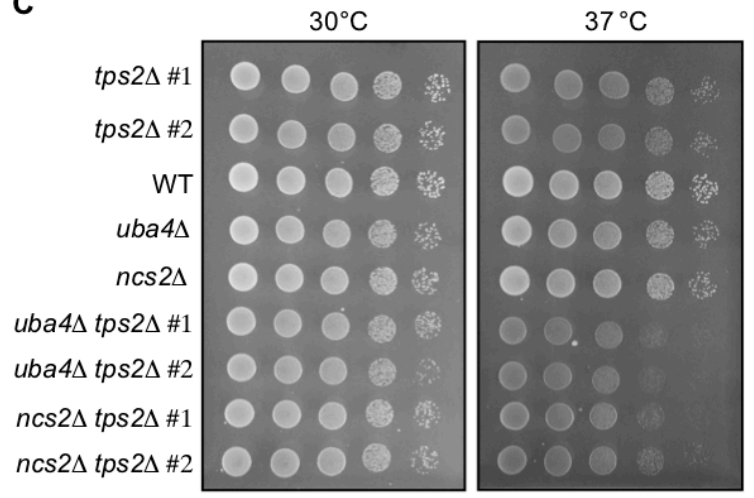

B

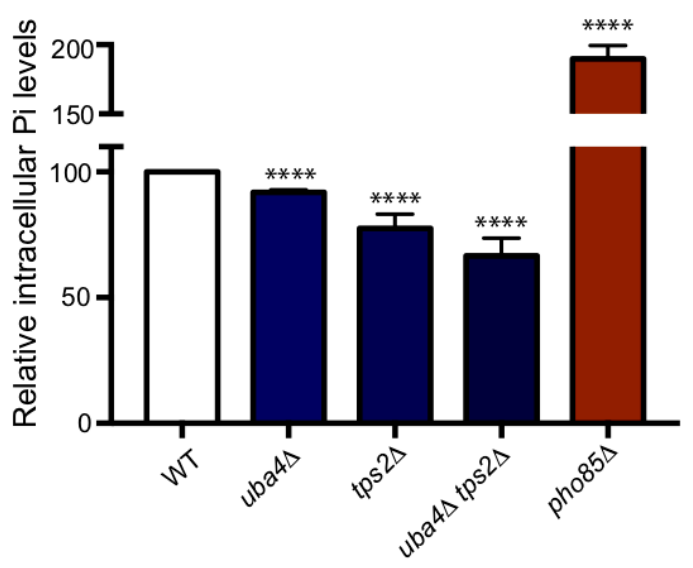

D

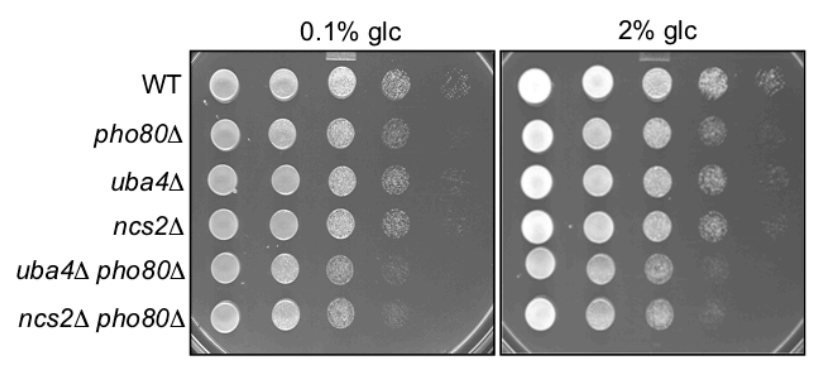

Figure 6

Figure 6: Trehalose synthesis associated phosphate release enables cells to maintain phosphate balance

(A) A schematic representation of the trehalose cycle, showing the routing of glucose-6-phospate towards trehalose and glycogen biosynthesis. Trehalose synthesis requires two glucose molecules, catalyzed by the Tps1 and Tps2 enzymes. The Tps2-mediated reaction synthesizes trehalose, and notably releases free $\mathrm{Pi}$.

955 (B) Intracellular Pi levels are maintained in tRNA thiolation mutant cells ( $u$ ba4 $4 \Delta$ ) by Tps2 activity. Free intracellular Pi levels were determined in WT, tRNA thiolation mutant (uba4 $\Delta$ ), tps $2 \Delta$, uba4 $\Delta$ tps $2 \Delta$ and pho85 cells grown in minimal media, by a colorimetric assay. Intracellular Pi levels in mutant $\mathrm{n}=2$ biological replicates with three technical replicates each. ${ }^{* * *} \mathrm{p}<0.0001$. Also see Figure S7A.

(C) Synthetic genetic interaction between TPS2 and tRNA thiolation genes (UBA4 and NCS2) at $37^{\circ}$ C. WT, tRNA thiolation mutants (uba4 $\Delta$ and $n c s 2 \Delta$ ), tps $2 \Delta, u b a 4 \Delta$ tps $2 \Delta$ and $n c s 2 \Delta$ tps $2 \Delta$ double mutant cells grown in minimal media were spotted on low Pi media agar plates with $2 \%$ glucose and incubated at $30^{\circ} \mathrm{C}$ and $37^{\circ} \mathrm{C}$. Also see Figure S7B.

964 (D) Synthetic genetic interaction between PHO80 and tRNA thiolation genes (UBA4 and NCS2). WT,

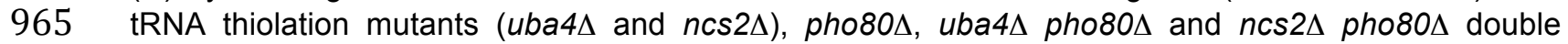
966 mutant cells grown in minimal media $(0.1 \%$ glucose $)$ were spotted on minimal media agar plates 967 ( $0.1 \%$ and $2 \%$ glucose $)$ and incubated at $30^{\circ} \mathrm{C}$. 

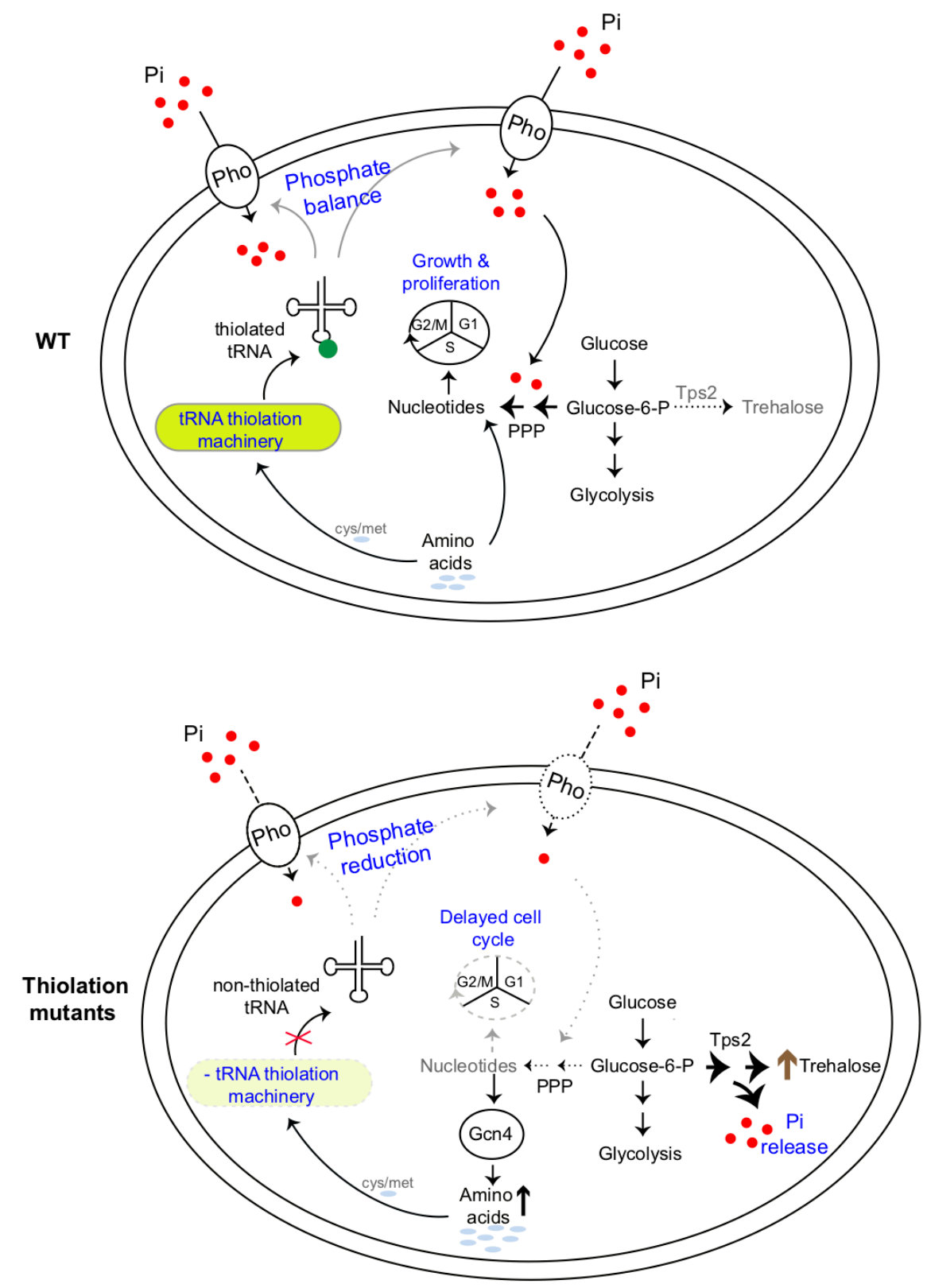

969 Figure 7: A simple model to illustrate the importance of tRNA thiolation in determining the metabolic state of the cell. WT cells (which have a functional tRNA thiolation machinery) sense and utilize amino acids (methionine and cysteine), and correspondingly have high amounts of thiolated tRNAs. In these cells, carbon flux coupled with amino acid utilization is towards nucleotide biosynthesis. Sufficient nucleotide levels support growth, and proper cell cycle progression, and reflect an overall 'growth' metabolic state. In tRNA thiolation mutants, the absence of tRNA thiolation machinery results in non-thiolated tRNAs, and carbon flux is driven away from nucleotide synthesis and towards storage carbohydrates, with a concurrent accumulation of amino acids. This metabolic rewiring in these mutants is due to reduced expression of genes involved in phosphate-responsive signalling pathway, which results in an effectively phosphate-limited state. Due to this, the thiolation mutants attempt to restore intracellular phosphate levels via Tps2-dependent trehalose synthesis, accompanied by phosphate release. Thus, despite the absence of carbon or nitrogen (amino acid) starvation, the tRNA thiolation mutants exhibit an overall metabolic signature of a 'starved state', with decreased nucleotide synthesis and delayed cell cycle progression.

983 Pho: phosphate transporters, Pi: inorganic phosphate, PPP: Pentose Phosphate Pathway, cys: 